\title{
Mantle source heterogeneity, magma generation and magmatic evolution at Terceira Island (Azores archipelago): Constraints from elemental and isotopic $(\mathrm{Sr}, \mathrm{Nd}, \mathrm{Hf}$, and $\mathrm{Pb}$ ) data
}

\author{
Pedro Madureira a,b,*, João Mata ${ }^{\text {, }}$, Nadine Mattielli ${ }^{\mathrm{d}}$, Gabriela Queiroz ${ }^{\mathrm{e}}$, Pedro Silva ${ }^{\mathrm{f}}$ \\ a Centro de Geofísica de Évora/Dep. Geociências da Universidade de Évora, R. Romão Ramalho, 59, 7000 Évora, Portugal \\ ${ }^{\mathrm{b}}$ Estrutura de Missão para os Assuntos do Mar, R. Costa Pinto, 165, 2770-047 Paço de Arcos, Portugal

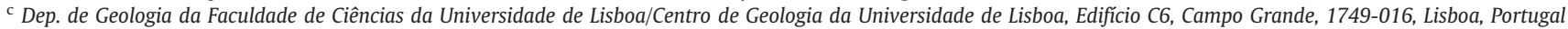 \\ ' Department of Earth and Environmental Sciences, DSTE, CP 160/02, Université Libre de Bruxelles, Avenue F.D. Roosevelt, 50, Brussels 1050, Belgium \\ e Centro de Vulcanologia e Avaliação de Riscos Geológicos da Universidade dos Açores, Edifício do Complexo Científico, $3^{\circ}$ Piso, Ala Sul, 9501-801, Ponta Delgada, Portugal \\ ${ }^{\mathrm{f}}$ ISEL/ADEC, R. Conselheiro Emídio Navarro, 1, 1959-007 Lisboa and Instituto Dom Luís, Universidade de Lisboa, Edifício C8, Lab. 8.1.70, Campo Grande, 1749-016, Lisboa, Portugal
}

\section{A R T I C L E I N F O}

\section{Article history:}

Received 14 November 2010

Accepted 12 July 2011

Available online 22 July 2011

\section{Keywords:}

Ocean Island Basalts

Small-scale mantle heterogeneity

Terceira Island

Azores

\begin{abstract}
A B S T R A C T
This work addresses the present-day ( $<100 \mathrm{ka})$ mantle heterogeneity in the Azores region through the study of two active volcanic systems from Terceira Island. Our study shows that mantle heterogeneities are detectable even when "coeval" volcanic systems (Santa Bárbara and Fissural) erupted less than $10 \mathrm{~km}$ away. These volcanic systems, respectively, reflect the influence of the Terceira and D. João de Castro Bank end-members defined by Beier et al. (2008) for the Terceira Rift. Santa Bárbara magmas are interpreted to be the result of mixing between a HIMU-type component, carried to the upper mantle by the Azores plume, and the regional depleted MORB magmas/source. Fissural lavas are characterized by higher $\mathrm{Ba} / \mathrm{Nb}$ and $\mathrm{Nb} / \mathrm{U}$ ratios and less radiogenic ${ }^{206} \mathrm{~Pb} /{ }^{204} \mathrm{~Pb},{ }^{143} \mathrm{Nd} /{ }^{144} \mathrm{Nd}$ and ${ }^{176} \mathrm{Hf} /{ }^{177} \mathrm{Hf}$, requiring the small contribution of delaminated sub-continental lithospheric mantle residing in the upper mantle. Published noble gas data on lavas from both volcanic systems also indicate the presence of a relatively undegassed component, which is interpreted as inherited from a lower mantle reservoir sampled by the ascending Azores plume. As inferred from trace and major elements, melting began in the garnet stability field, while magma extraction occurred within the spinel zone. The intra-volcanic system's chemical heterogeneity is mainly explained by variable proportions of the above-mentioned local end-members and by crystal fractionation processes.
\end{abstract}

(C) 2011 Elsevier B.V. All rights reserved.

\section{Introduction}

It has been demonstrated that the Earth's mantle is heterogeneous (e.g., Gast et al., 1964) at several scales, from $\mathrm{mm}^{3}$ to $10^{4} \mathrm{~km}^{3}$. This claim is supported by: 1) the study of mantle xenoliths (e.g., Bonadiman et al., 2005; Harte, 1987; Mattielli et al., 1999) and of outcropping mantle portions (e.g., Brunelli and Seyler, 2010; Reisberg and Zindler, 1986) preserving evidence for millimeter- to meter-scale heterogeneities caused by metasomatic processes; 2) the significant heterogeneity depicted by oceanic basalts (e.g., Hofmann, 2003); and 3 ) the existence of large mantle domains with peculiar characteristics, as shown by the Dupal anomaly (Dupré and Allègre, 1983; Hart, 1984).

\footnotetext{
* Corresponding author at: Centro de Geofísica de Évora/Dep. Geociências da Universidade de Évora, R. Romão Ramalho, 59, 7000 Évora, Portugal. Tel.: + 351 266745301; fax: + 351266745397.

E-mail addresses: pedro@uevora.pt (P. Madureira),jmata@fc.ul.pt (J. Mata), nmattiel@ulb.ac.be (N. Mattielli), maria.gp.queiroz@azores.gov.pt (G. Queiroz), pmfsilva@fc.ul.pt (P. Silva).
}

The Azores islands, located on the Azores Plateau and close to the Mid-Atlantic Ridge, are characterized by significant elemental and isotopic variations, which are evident either from the study of a single island (Terceira: Dupré et al., 1982; S. Miguel: Beier et al., 2007; Haase and Beier, 2003; S. Jorge: Hildenbrand et al., 2008; Millet et al., 2009) or from regional studies (e.g., Beier et al., 2008, 2010; Moreira et al., 1999; White et al., 1979; Widom and Shirey, 1996). At Northeast the Azores Plateau is bordered by the Terceira Rift (Fig. 1A), which has been interpreted as an intra-oceanic spreading system (see Vogt and Jung, 2004 and references therein). Beier et al. (2008) identified the presence of several distinct compositional end-members along this structure, providing evidence for significant mantle source heterogeneity and focused magmatism with no significant melt or source interaction between different volcanic systems.

Although some authors have asserted that the Azores resulted from an anomalously volatile-enriched upper mantle domain (e.g. Bonatti, 1990), the conjunction of noble gas signatures with data from seismic tomography endorses the idea of a mantle plume influence on the Azores magmatism (Jean-Baptiste et al., 2009; Madureira et al. 2005; Moreira et al., 1999; Silveira et al., 2006, 2010). However, the 
A

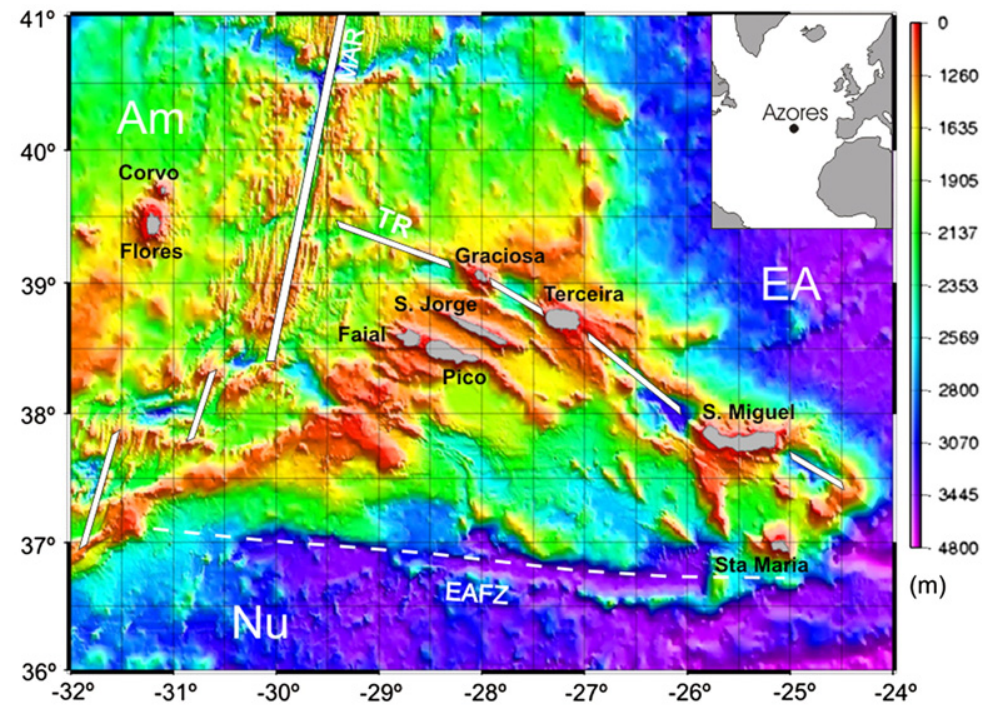

B

\section{Volcanic system}

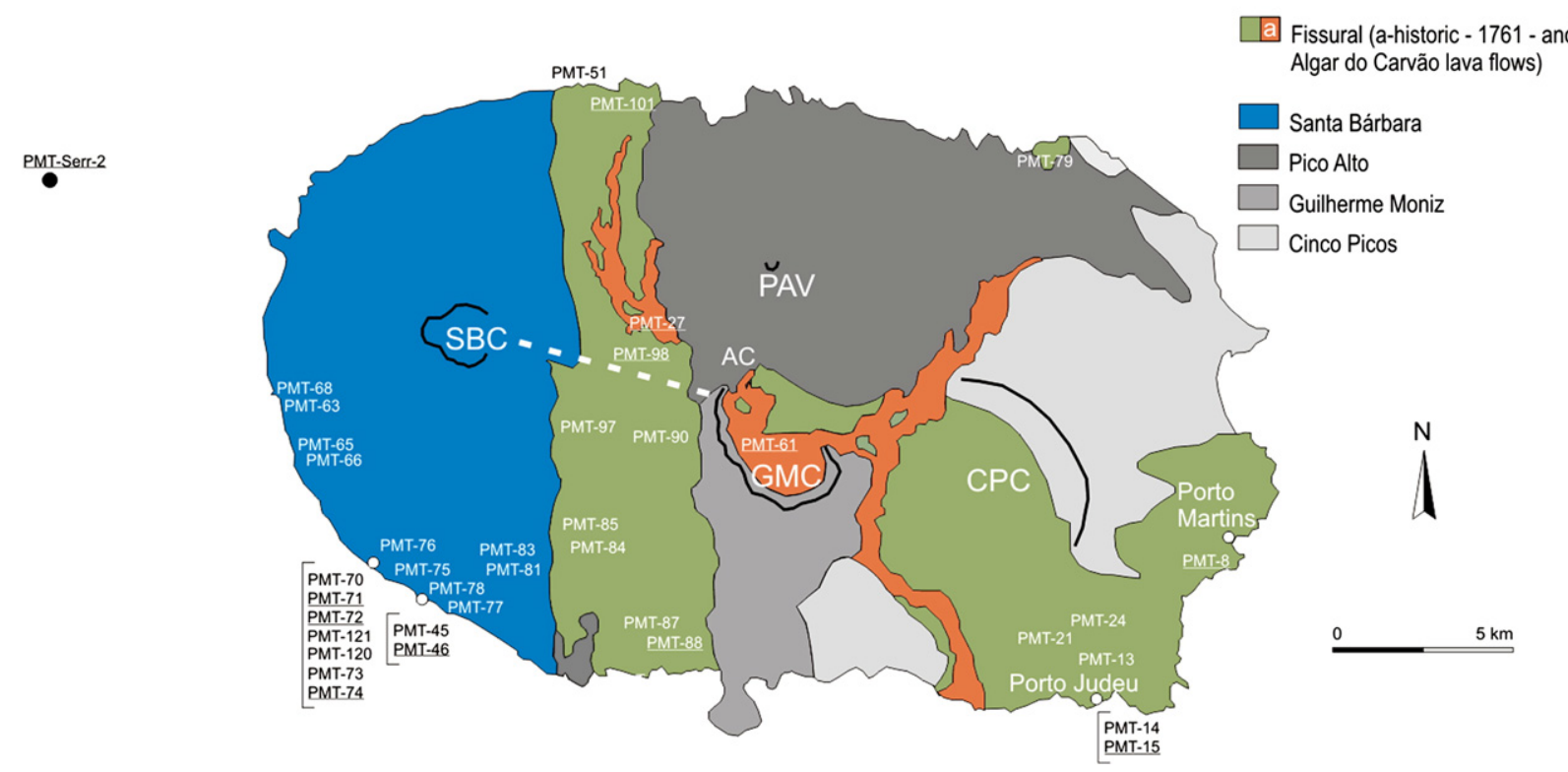

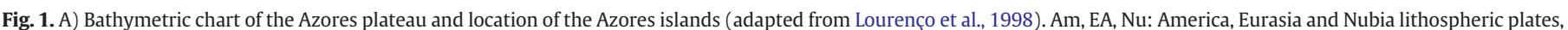

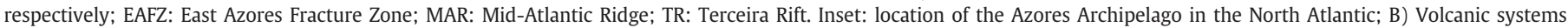

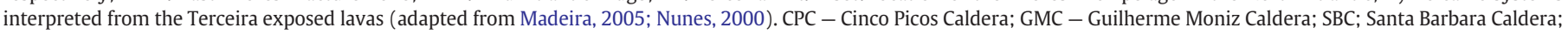

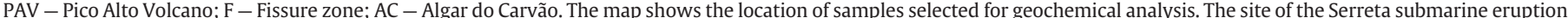

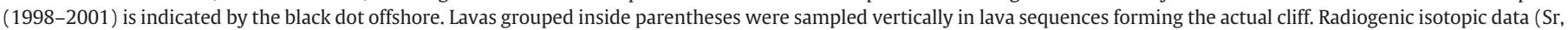

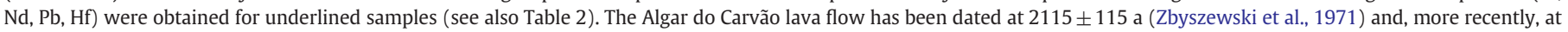
$1730 \pm 70$ a (Forjaz et al., 2004).

relative role of a mantle plume and shallow level-reservoirs as the cause for such variability has been the locus of an intense debate (e.g., Millet et al., 2009; Moreira et al., 1999; Widom et al., 1997).

Through a finite-frequency seismic tomography study, Yang et al. (2006) demonstrated that the low-velocity anomaly is centered NE of Terceira Island and is deflected to the SE by asthenospheric flow. This and the fact that some of the more unradiogenic He ratios for the Azores islands were obtained in olivine phenocrysts from Terceira, where ${ }^{21} \mathrm{Ne} /{ }^{22} \mathrm{Ne}_{\text {corr }}=0.052$ (Madureira et al., 2005), suggests that the Azores mantle plume can be particularly well sampled by Terceira lavas.

In this study, elemental and isotope data ( $\mathrm{Sr}, \mathrm{Nd}, \mathrm{Pb}$, and $\mathrm{Hf}$ ) were obtained for Terceira's mafic lavas. They were collected from the two main active volcanic systems linked to the Santa Bárbara central volcano and to the fissure zone, the latter being considered the emerged record of the Terceira Rift. Based on available $\mathrm{K} / \mathrm{Ar}$ and $\mathrm{Ar} / \mathrm{Ar}$ ages (Calvert et al., 2006; Féraud et al., 1980) the sampled lavas are expected to be younger than $100 \mathrm{kyr}$. The obtained data will be used to address the following issues: 1 ) to confirm and better constrain the occurrence of intra-Terceira Island heterogeneity referred by Dupré et al. (1982);2) to address intra-volcanic system chemical variability; 3 ) to compare the Terceira isotopic signatures with those described by Beier et al. (2008) for the Terceira Rift as a whole; 4) to constrain the melting processes and the depth of magma extraction; and 5) to discuss the origin of the main contributors to the Terceira source(s) and their importance in the context of the Azores.

\section{Geological background and sampling strategy}

The Azores archipelago is located in the North Atlantic $\left(37^{\circ}-40^{\circ} \mathrm{N}\right)$ near the RRR-type triple junction where the Eurasia, Nubia (Africa) 
and America plates meet (Fig. 1A). The archipelago is composed of nine volcanic islands emerging from the Azores Plateau $\left(400,000 \mathrm{~km}^{2}\right.$ of elevated oceanic crust, underlined by $2000 \mathrm{~m}$ isobaths; Lourenço et al., 1998) that extend to both sides of the Mid-Atlantic Ridge (MAR). To the east of the MAR, the northern boundary of the Azores Plateau is underlain by a $550 \mathrm{~km}$ long hyper-slow $(\sim 4 \mathrm{~mm} / \mathrm{a})$ rift system known as the Terceira Rift. The onset of the extensional regime in the Azores domain may have begun 20 Ma ago (Luís and Miranda, 2008) with focused spreading along the Terceira Rift occurring essentially in the last $1 \mathrm{Ma}$ (Vogt and Jung, 2004). Its present-day extensional regime is confirmed by GPS and laser measurements on the islands (e.g., Miranda et al., 1998). The islands of S. Miguel, Terceira and Graciosa, as well as the submarine D. João de Castro Bank are all located along this rift and separated by deep avolcanic zones that reach $3200 \mathrm{~m}$ bsl at the Hirondelle basin (Beier et al., 2008; Lourenço, 2007; Vogt and Jung, 2004). The Terceira Rift is considered the boundary between the Eurasia and Nubia lithospheric plates, which was previously located to the southwest as suggested by the elongation of $\mathrm{S}$. Jorge Island, the Faial-Pico alignment and other elongated ridges with similar orientations (Vogt and Jung, 2004; see Fig. 1A).

The morphology and volcano-stratigraphy of Terceira Island indicate the existence of five main volcanic systems (Fig. 1B; Madeira, 2005; Nunes, 2000): Cinco Picos, Guilherme Moniz, Pico Alto, Santa Bárbara and one associated to the fissure zone (hereafter referred as the Fissural volcanic system). The latter connects the Santa Bárbara and Guilherme Moniz calderas and is believed to extend toward the SE coast of Terceira Island (Self, 1976). Cinco Picos comprises the oldest volcanic center (older than $370 \mathrm{ka}$; Calvert et al., 2006) and is currently dominated by the largest caldera of the Azores archipelago ( $\sim 7 \mathrm{~km}$ diameter), which is partially filled with lavas erupted from the fissure zone. The Fissural, Santa Bárbara and Pico Alto systems are considered volcanically active, but for the latter only highly evolved lavas and pyroclastic deposits (pantellerites and comendites) outcrop at the surface (Gertisser et al., 2010; Mungall and Martin, 1995; Self and Gunn, 1976). The eruption of similar high-silica lavas also occurred during the last stage of Santa Bárbara's evolution with the formation of $\sim 80$ lava domes (Madeira, 2005; Self and Gunn, 1976). For this volcano, Calvert et al. (2006) estimated the beginning of subaerial activity within the past $100 \mathrm{kyr}$.

For Terceira Island, Self (1976) reported the occurrence of 116 eruptions before the generation of a 23,000 year old ignimbrite. However, in historical times only three basaltic eruptions occurred restricted to the fissure zone. The last eruptions (1998-2001) took place offshore $10 \mathrm{~km}$ to the west of the island at the Serreta submarine ridge, where a previous eruption occurred in 1867 closer to the island (Gaspar et al., 2003). Our basaltic sample, PMT-Serr-2, was collected in April 1999 during one of the major submarine volcanic activity periods that brought lava balloons to the sea surface. This study is focused on mafic lavas from the Fissural and Santa Bárbara volcanic systems (See Fig. 1B for location) to assess the "present-day" Terceira mantle source(s) and magma formation processes.

\section{Analytical procedure}

Whole-rock analyses of major and trace elements were performed in Canada by Activation Laboratories using ICP-OES and ICP-MS, respectively. Alkaline dissolution with lithium metaborate/tetraborate followed by nitric acid was used for the analyses, except for $\mathrm{Ni}, \mathrm{Cu}, \mathrm{Zn}$ and $\mathrm{Cd}$ determination, for which multi-acid digestion (hydrofluoric followed by a mixture of nitric and perchloric acids) was carried out. Reproducibility was generally better than $5 \%$ for both major and trace elements and can be better evaluated at Supplementary Material (Table S1) where we also present analyses of standard reference materials (BIR-1,W-2a and DNC-1). More information on the analytical procedure can be found at http://www.actlabs.com.

The isotopic analyses of 12 representative samples from the Fissural and Santa Bárbara systems were performed at the Department of Earth and Environmental Sciences of the Université Libre de Bruxelles (Nd, Hf and $\mathrm{Pb}$ isotopes) and at Pacific Centre for Isotopic and Geochemical Research at the University of British Columbia ( $\mathrm{Sr}$ isotopes). The samples were dried at $40{ }^{\circ} \mathrm{C}$ and crushed in an agate mortar under an extracting hood. The entire acid digestion process and the analyte separation on ion-exchange resin were performed in a class 100laminar air flow cabinet. About $200 \mathrm{mg}$ of powder was dissolved in closed Savilex ${ }^{\circledR}$ beakers using $\mathrm{HF}-\mathrm{HNO}_{3}\left(130^{\circ} \mathrm{C}, 48 \mathrm{~h}\right)$ followed by evaporation, the addition of $6 \mathrm{~N} \mathrm{HCl}$, a second evaporation and final dissolution in $0.5 \mathrm{~N} \mathrm{HBr}$. Lead separation was performed using successive acid elution on an anionic resin (AG1-X8) column (for further details, see Weis et al., 2006). Sr, REE and Hf were isolated using a column loaded with $18.5 \mathrm{ml}$ of Dowex AG50W-X8 100-200 mesh cation exchange resin. Nd was recovered by loading the REE fraction onto a column with HDEHP-coated Teflon. Hafnium was separated following the slightly modified two-column procedure described in Blichert-Toft et al. (1997), involving anion and cation exchange columns (see Mattielli et al., 2002; Weis et al., 2006). Measurements of the total procedural $\mathrm{Pb}, \mathrm{Nd}, \mathrm{Sr}$ and Hf blanks gave ca. $10 \mathrm{pg}, 28 \mathrm{pg}$, $400 \mathrm{pg}$ and $23 \mathrm{pg}$, respectively. The isotopic compositions of $\mathrm{Pb}, \mathrm{Nd}$ and Hf were determined on a Nu plasma MC-ICP-MS (multiple collector inductively coupled plasma mass spectrometer). At the beginning of every analytical sessions, collected analyte ( $\mathrm{Pb}$ or $\mathrm{Nd}$ or $\mathrm{Hf}$ ) elution fractions were re-dissolved in $100 \mu \mathrm{l}$ of concentrated $\mathrm{HNO}_{3}$, evaporated and finally dissolved in $1.5 \mathrm{ml}$ of $\mathrm{HNO}_{3} 0.05 \mathrm{~N}$. Tl was added to each $\mathrm{Pb}$ sample and standard to control instrumental mass fractionation. Solutions were prepared to obtain a $\mathrm{Pb} / \mathrm{Tl}$ rate of 4 or 5 and a signal of $100 \mathrm{mV}$ in the axial collector $\left({ }^{204} \mathrm{~Pb}\right)$ as well as to reach the $\mathrm{Pb}$ and $\mathrm{Tl}$ concentrations of the standard $(200 \mathrm{ppb}$ of $\mathrm{Pb}$ and $50 \mathrm{ppb}$ of $\mathrm{Tl}$ ). Standards were systematically run between every two samples to monitor the instrumental mass bias during the analysis session, resulting in ${ }^{143} \mathrm{Nd} /{ }^{144} \mathrm{Nd}=0.511960 \pm 9(2 \sigma, n=11)$ for the Rennes standard, solution (see Chauvel and Blichert-Toft, 2001), and ${ }^{176} \mathrm{Hf} /{ }^{177} \mathrm{Hf}=0.282149 \pm 11(2 \sigma, n=31)$ for the JMC475 Hf standard. All $\mathrm{Pb}$ measurements were automatically corrected according to the $\mathrm{Tl}$ mass fractionation and then by using the standard bracketing method with the recommended values of Galer and Abouchami (1998) and Abouchami et al. (2000). The Nd and Hf isotopic measurements were internally normalized to ${ }^{146} \mathrm{Nd} /{ }^{144} \mathrm{Nd}=0.7219$ and ${ }^{179} \mathrm{Hf} /{ }^{177} \mathrm{Hf}=$ 0.7325 , respectively. The $\mathrm{Sr}$ isotopic ratios were analyzed on a Finnigan Triton Thermo-Ionization Mass Spectrometer (TIMS). The NBS987 $\mathrm{Sr}$ standard was run after every 2 samples, giving a mean value of ${ }^{87} \mathrm{Sr} /{ }^{86} \mathrm{Sr}=0.710260 \pm 25(2 \sigma)$ for 6 measurements. The raw data were corrected for mass fractionation using ${ }^{86} \mathrm{Sr} /{ }^{88} \mathrm{Sr}=0.1194$.

In our study, each session of analyses starts by a "batch run" of 10 analyses of the standard solutions in order to monitor the optimization (stability and sensitivity) of the machine. In addition, during this batch run, the reproducibility and accuracy of the values were checked. The quality of our analytical procedure (i.e. chemical preparation procedures and isotopic measurements), and especially the reproducibility of isotopic measurements, can be assessed by sample replicate and duplicate analyses, which is better than $49 \mathrm{ppm}, 19 \mathrm{ppm}, 25 \mathrm{ppm}$ for ${ }^{143} \mathrm{Nd} /{ }^{144} \mathrm{Nd}$, ${ }^{176} \mathrm{Hf} /{ }^{177} \mathrm{Hf},{ }^{87} \mathrm{Sr} /{ }^{86} \mathrm{Sr}$, respectively. For ${ }^{206} \mathrm{~Pb} /{ }^{204} \mathrm{~Pb}$, ${ }^{207} \mathrm{~Pb} /{ }^{204} \mathrm{~Pb}$, and ${ }^{208} \mathrm{~Pb} /{ }^{204} \mathrm{~Pb}$ the external reproducibility is $\sim 180 \mathrm{ppm}$. Additional information on the analytical procedures, as well as on the usual accuracy and reproducibility obtained at the Université Libre de Bruxelles and the University of British Columbia, can be found in Weis et al. (2006).

Minerals from representative samples were analyzed with a JEOL JCXA 733X electron microprobe at Centro de Geologia da Universidade de Lisboa (CeGUL). The microprobe operated with a 5 - $\mu$ m beam diameter, an accelerating voltage of $15 \mathrm{kV}$ (18 kV for opaque minerals) and a beam current of $25 \mathrm{nA}$. Natural mineral and pure metal $(\mathrm{Cr}, \mathrm{V}$ and $\mathrm{Ni}$ ) standards were used. Precision, as indicated by replicate determinations on an in-house standard, was better than $2 \%$ for the major elements. 


\section{Results}

\subsection{Petrography and chemical composition of the main mineral phases}

The textures of mafic lavas from the Santa Bárbara and Fissural volcanic systems are predominantly porphyritic to seriate with major phenocryst phases (olivine, clinopyroxene and plagioclase) invariably surrounded by a microcrystalline matrix dominated by plagioclase and opaque minerals (Fig. 2). Opaque microphenocrysts are also present in most Santa Bárbara lavas. For the less evolved lavas ( $\mathrm{Mg \#}>68$ ), our petrographic observations indicate olivine as the liquidus phase, which was early joined by clinopyroxene and then plagioclase in the more evolved samples. The proportion of phenocrysts in Fissural lavas ranges between 1 and 15\%, significantly less than the maximum of $25-30 \%$ (of the rock volume) observed for Santa Bárbara lavas.

Concerning Fissural lavas, plagioclase phenocrysts are nearly absent in rocks outcropping in the eastern area of Terceira Island and are considerably more abundant in lavas that erupted between the Santa Bárbara and Guilherme Moniz calderas, where more evolved lavas occur. This strongly suggests the presence of more mature magma chambers in the central part of the island.

In Santa Bárbara lavas, plagioclase is always the dominant phenocryst commonly forming glomeroporphyritic clusters. Olivine phenocrysts are poorly represented and often exhibit iddingsite along their rims and/or intra-granular fractures. The rarity of olivine is considered as a result of the evolved character of lavas $(\mathrm{Mg} \#<48)$ in this volcanic system. Biotite and/or kaersutite crystals can also be observed in some Santa Bárbara lavas occurring both as a matrix phase and as filling vesicles.

Representative chemical analysis of the major phenocryst phases in Fissural and Santa Bárbara lavas can be found in Supplementary Material (Table S2).

\subsubsection{Olivine}

Olivine phenocrysts with the highest forsterite (Fo) content were analyzed in Fissural lavas (88-90\% Fo) sampled in the eastern area of Terceira Island, which also display higher measured $\mathrm{NiO}$ concentrations (0.3-0.4 wt.\%; Supplementary Material, Table S2A). Forsterite ranges from $90 \%$ to $72 \%$ in phenocryst cores from these lavas and from $85 \%$ to $56 \%$ in olivine phenocrysts from the Santa Bárbara volcanic system.

For both volcanic systems there is a crude positive co-variation between the $\mathrm{CaO}$ concentration ( 0.15 to $0.38 \mathrm{wt} . \%)$ and \%Fo in olivine cores (not shown). If the crystallization of the higher magnesian olivines occurred at deeper levels, this would be contrary to what is expected from the inverse correlation between pressure and the $\mathrm{D}_{\mathrm{Ca}}^{\mathrm{Oliv} / \mathrm{liq}}$ partition coefficient (Hirschmann and Ghiorso, 1994). According to petrographic observations, we explain such correlation as the result of $\mathrm{CaO}$ depletion stemming from coeval clinopyroxene crystallization (Libourel, 1999; Jurewicz and Watson, 1988). For some lavas, zoned compositional patterns display a large central plateau characterized by relatively high Fo contents, which is rimmed by a thin border marked by significantly lower $\mathrm{MgO}$ contents. This is interpreted as reflecting rapid $\mathrm{MgO}$ depletion in magmas as a consequence of sudden microlyte nucleation during mesostasis crystallization (e.g., Hildner et al., 2011).

\subsubsection{Clinopyroxene}

Clinopyroxene is a major phase in Terceira lavas, occurring as phenocrysts, microphenocrysts and matrix crystals. Ubiquitous phenocryst compositional zoning can be readily interpreted from crossed nicols observations and, in most cases, also from the darker brown color of the outer crystal rim. Terceira pyroxenes are dominated by quadrilateral components and, following the classification of Morimoto et al. (1988), can be considered as diopsides and augites. The highest $\mathrm{Mg} \#$ of clinopyroxene cores is identical to that obtained for olivine, but their range is smaller than what was observed for olivine phenocrysts (Mg\# range from 0.75 to 0.90 for Fissural lavas and from 0.73 to 0.80 for
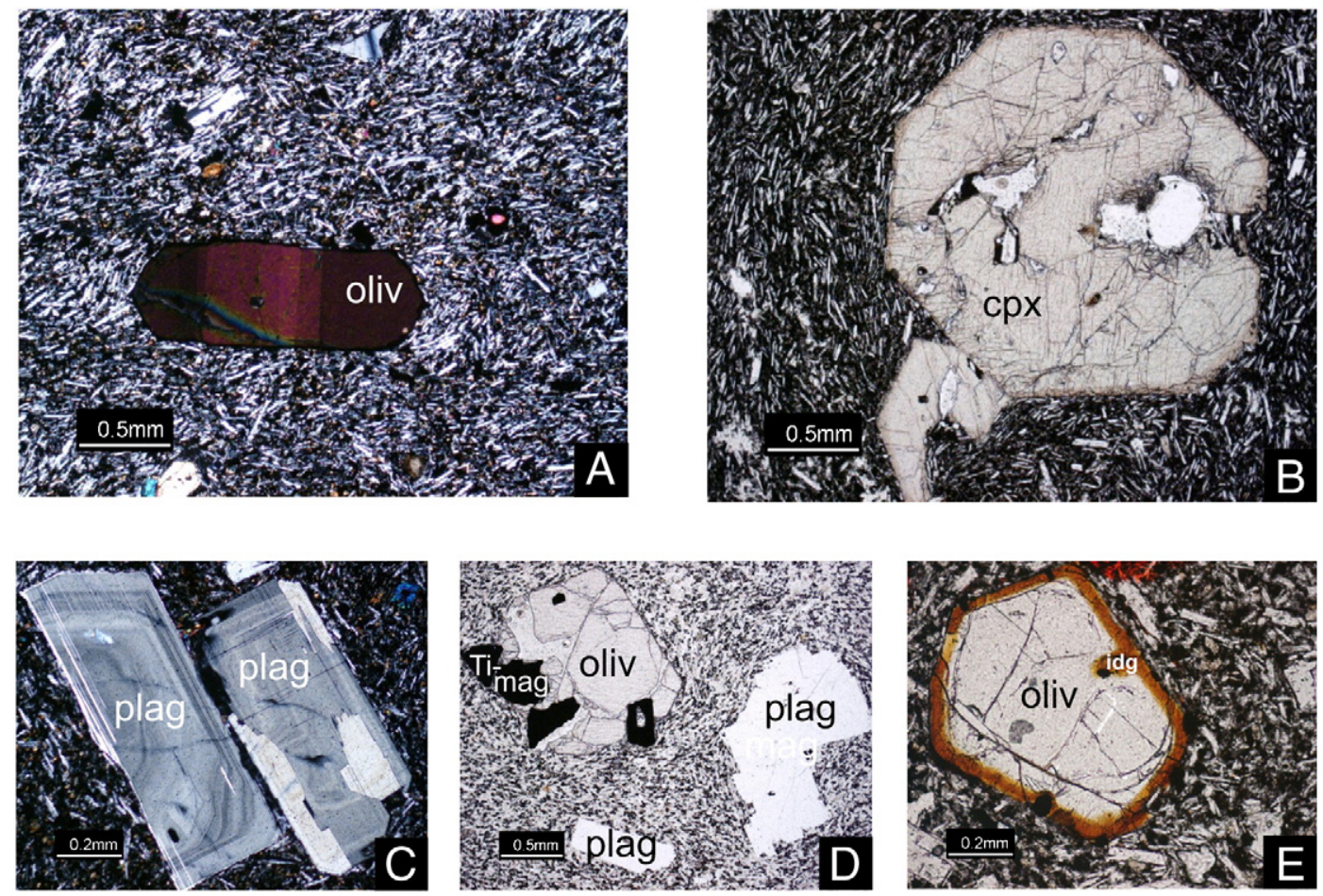

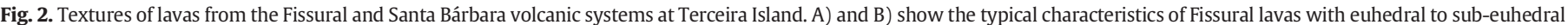

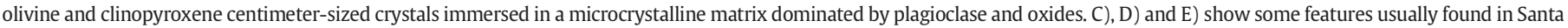

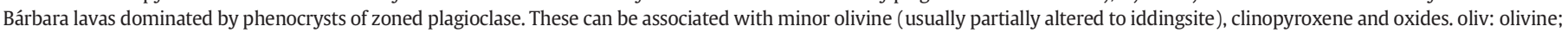
cpx: clinopyroxene; plag: plagioclase; Ti-mag: titanomagnetite; idg: iddingsite. 
Santa Bárbara lavas; Supplementary Material, Table S2B). We consider the overlapping of the Mg\# for the most magnesium pyroxenes and olivines as the result of equilibrium crystallization between those two phases given the similarity between their $K_{D}{ }^{\text {min/liq }} \mathrm{Fe} / \mathrm{Mg}$ (see Tappe, 2004). Fissural and Santa Bárbara clinopyroxene phenocrysts are distinguishable by higher $\mathrm{Cr}$ concentrations (up to $1.2 \mathrm{wt} . \%$ ) and $\mathrm{Al} / \mathrm{Ti}$ ratios (4.57) of the former compared with those observed for Santa Bárbara pyroxenes $\left(\mathrm{Cr}_{2} \mathrm{O}_{3}\right.$ up to 0.6 wt.\%; $\mathrm{Al} / \mathrm{Ti}$ up to 2.86$)$, reflecting the less evolved character of the Fissural lavas.

\subsubsection{Plagioclase}

Plagioclase is the dominant phenocryst phase of Santa Bárbara lavas and is also important in the most evolved Fissural lavas sampled in the central area of Terceira Island. The analyzed phenocrysts display a wide range in composition from $40 \%$ to $85 \%$ An (Supplementary Material, Table S2C). Groundmass plagioclase laths extend the compositional range down to $30 \%$ An. Multiple and complex zoning surrounding resorbed cores is a common feature in Santa Bárbara plagioclase phenocrysts. Several profiles along Santa Bárbara plagioclase phenocrysts also display oscillatory zoning, which can be interpreted as a probable result of mixing between melt fractions with different evolutionary stages.

\subsubsection{Oxides}

Opaque minerals can be found as inclusions in phenocryst phases, as (micro)phenocrysts in Santa Bárbara lavas and as crystals from the microcrystalline matrix. Inclusions and (micro)phenocrysts are represented by minerals from the spinel group, mainly chromite and titanomagnetite (Supplementary Material, Table S2D). Ilmenite is the dominant oxide in the microcrystaline matrix both in Fissural and in Santa Bárbara lavas but can also occur as (micro)phenocrysts in Santa Bárbara lavas or as exsolution lamellae in titanomagnetite phenocrysts.

\subsection{Whole-rock chemistry}

Whole-rock data were obtained from unaltered samples, usually with less than $10 \%$ of phenocrysts to be representative of magmatic liquid compositions (Table 1). However, some Santa Bárbara lavas display higher volume proportions of plagioclase phenocrysts (up to $35 \%$ ), which suggests plagioclase accumulation. This interpretation is strengthened by a positive correlation between the $\mathrm{Eu} / \mathrm{Eu}^{*}$ and $\mathrm{Sr} / \mathrm{Nd}$ ratios (not shown), and, accordingly, those samples were omitted in this discussion and did not contribute to our conclusions.

\subsubsection{Major elements}

Similarly to other Azores Islands and OIB, the elemental data obtained for the Terceira Fissural and Santa Bárbara lavas are distinct from Atlantic MORB. Most primitive lavas (here considered to have $\mathrm{MgO} \geq 8$ wt.\%) present higher $\mathrm{TiO}_{2}$ and $\mathrm{P}_{2} \mathrm{O}_{5}$ contents than typical N-MORB (2.3-3.0 and 0.35-0.86 wt.\% versus $1.19-1.77$ and $0.10-$ 0.16 wt.\%, respectively; e.g., Sridhar and Ray, 2003) and $\mathrm{Na}_{2} \mathrm{O} / \mathrm{K}_{2} \mathrm{O}$ ratios (2.8 to 4.3) similar to the values reported for other Atlantic islands such as Madeira, Saint Helena, Cape Verde and Canary (e.g., Chaffey et al., 1989; Doucelance et al., 2003; Gurenko et al., 2006; Holm et al., 2006; Martins et al., 2010; Mata et al., 1998). This range of values was also found in most of the Azores islands with the notable exception of eastern $\mathrm{S}$. Miguel, which is characterized by significantly lower $\mathrm{Na}_{2} \mathrm{O}$ / $\mathrm{K}_{2} \mathrm{O}$ ratios (1.3-1.9) in accordance with its potassic character (e.g., Beier et al., 2007; Flower et al., 1976; França et al., 2006; Schminke, 1973; Widom et al., 1997).

In the TAS (Total Alkali-Silica) diagram, most Terceira samples are plotted above the line proposed by Irvine and Baragar (1971) to separate the alkaline and subalkaline compositional fields (Fig. 3). Despite their alkaline affinity, basaltic samples are only slightly nepheline normative (less than 3\%), or even hypersthene normative (see also White et al., 1979), in contrast with the more $\mathrm{SiO}_{2}$ - undersaturated compositions found for most of the Azores archipelago (e.g., Davies et al., 1989; Schminke, 1973). This more $\mathrm{SiO}_{2}$-saturated tendency of Terceira lavas partially explains the common occurrence of ilmenite, a mineral phase whose crystallization is strongly inhibited by the significant $\mathrm{Ti}$ incorporation in clinopyroxene in $\mathrm{SiO}_{2}$-undersaturated rocks (e.g., Toplis and Carroll, 1995). The high ilmenite abundance allows the distinction of Terceira lavas even from moderate alkaline lavas such as those of Madeira Island (see Mata and Munhá, 2004).

\subsubsection{Trace elements}

Terceira most primitive lavas ( $\mathrm{MgO} \geq 8$ wt.\%) are characterized by fractionated REE patterns with primitive mantle normalized $\mathrm{La} / \mathrm{Yb}$ and $\mathrm{Tb} / \mathrm{Yb}$ ratios ranging from 7.4 to 11.5 and 2.2 to 2.7 , respectively. Variable $\mathrm{La} / \mathrm{Yb}$ and $\mathrm{Tb} / \mathrm{Yb}$ ratios for basaltic samples are suggestive of differences in the degree of partial melting and in the amount of residual garnet on magma genesis (e.g., George and Rogers, 2002). For Terceira Island, the presence of garnet in the mantle source is also suggested by the low concentrations of HREE (Yb: 1.87-2.67 ppm; Lu: 0.25-0.37 ppm) in the most primitive lavas when compared with the mean values obtained for N-MORB (3.05 ppm and 2.37 ppm respectively; Sun and McDonough, 1989). Moreover, the presence of residual garnet is supported by the ${ }^{230}$ Th excesses up to $26 \%$ over ${ }^{238} \mathrm{U}$ determined by Turner et al. (1997) for Terceira lavas.

Looking at primitive mantle normalized diagrams, it is possible to recognize for both systems the enrichment of $\mathrm{Nb}$ and Ta relative to LREE and LILE and the presence of $\mathrm{K}$ negative anomalies (Fig. 4). However, Fissural lavas are almost ubiquitously characterized by a positive Ba anomaly that is never observed in Santa Bárbara lavas, suggesting that magmas from these two volcanic systems correspond to distinct magmatic series. This assertion is also strengthened by the different incompatible trace elements and isotopic ratios found for Fissural and Santa Bárbara lavas (see Fig. 5 and Section 4.3). Santa Bárbara lavas present trace element patterns similar to those described for HIMU-type basalts, while Fissural samples, with higher $\mathrm{Ba} / \mathrm{Nb}, \mathrm{Ba} / \mathrm{Th}$ and $\mathrm{Rb} / \mathrm{Th}$ ratios, are more akin to EM I-type magmas (see insets in Fig. 4 and Weaver, 1991).

The submarine lavas erupted in 1999 along the Serreta ridge, at the prolongation of the fissure zone, represents a special case since they display trace element characteristics similar to those erupted from the Santa Bárbara central volcano (Fig. 5). This suggests that the Santa Bárbara mantle source is presently being sampled by eruptive events at the fissure zone.

\subsection{Isotopic data}

The $\mathrm{Sr}, \mathrm{Nd}, \mathrm{Pb}$ and $\mathrm{Hf}$ isotopic data obtained for some Fissural and Santa Bárbara lavas are summarized in Table 2. Considering the very young age of the studied lavas ( $<0.1 \mathrm{Ma}$; Calvert et al., 2006) and the very long half-life of the radioactive parent isotopes $\left(>0.703 \times 10^{9} \mathrm{y}\right)$, the analyzed values are equal to the calculated initial isotopic ratios.

In the $\mathrm{Sr}-\mathrm{Nd}$ isotope diagram, Terceira samples are plotted in the mantle array in the vicinity of the HIMU and DMM components (Fig. 6A). They display a restricted range of isotopic ratios $\left({ }^{87} \mathrm{Sr} /{ }^{86} \mathrm{Sr}\right.$ : $0.703425-0.703596 ;{ }^{143} \mathrm{Nd} /{ }^{144} \mathrm{Nd}: 0.512908-0.512980 ; \varepsilon \mathrm{Nd}:+5.3$ to +6.7 ), showing evidence for the time-integrated depletion of $\mathrm{Rb}$ and $\mathrm{Nd}$ relative to the more compatible $\mathrm{Sr}$ and $\mathrm{Sm}$, respectively.

In $\mathrm{Pb}$ isotope diagrams (Fig. $6 \mathrm{~B}$ and $\mathrm{C}$ ), the studied Terceira samples plot slightly beneath the NHRL ( $\Delta 7 / 4$ down to $-5.80 ; \Delta 8 / 4$ down to -53.92) in agreement with previous data (e.g., Davies et al., 1989; Turner et al., 1997). At the scale of the island, it is evident that Fissural and Santa Bárbara lavas present different $\mathrm{Pb}$ isotope signatures (see also Dupré et al., 1982) with the latter being more radiogenic $\left({ }^{206} \mathrm{~Pb} /{ }^{204} \mathrm{~Pb}\right.$ from 19.928 to $19.992 ;{ }^{207} \mathrm{~Pb} /{ }^{204} \mathrm{~Pb}$ from 15.614 to $15.620 ;{ }^{208} \mathrm{~Pb} /{ }^{204} \mathrm{~Pb}$ from 39.226 to 39.266 ) than Fissural lavas $\left({ }^{206} \mathrm{~Pb} /{ }^{204} \mathrm{~Pb}\right.$ from 19.339 to 19.846; ${ }^{207} \mathrm{~Pb} /{ }^{204} \mathrm{~Pb}$ from 15.529 to $15.617 ;{ }^{208} \mathrm{~Pb} /{ }^{204} \mathrm{~Pb}$ from 38.872 to 39.204). Moreover, looking at the fissure zone, there is a tendency for a 
Table 1

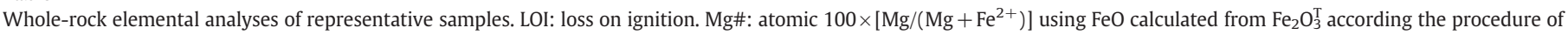

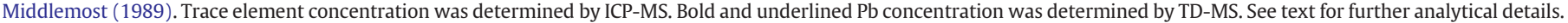
Lithotype determined according Le Bas et al. (1986).

\begin{tabular}{|c|c|c|c|c|c|c|c|c|c|c|c|c|}
\hline \multirow{2}{*}{$\frac{\text { Volc. Syst. }}{\text { Sample }}$} & \multicolumn{11}{|l|}{ Fissural } & \multirow{2}{*}{$\frac{\text { Serreta Ridge }}{\text { PMT-Serr-2 }}$} \\
\hline & \multirow{2}{*}{$\frac{\text { PMT-8 }}{\text { Basalt }}$} & \multirow{2}{*}{$\frac{\text { PMT-14 }}{\text { Basalt }}$} & \multirow{2}{*}{$\frac{\text { PMT-15 }}{\text { Basalt }}$} & PMT-21 & PMT-27 & PMT-61 & PMT-79 & PMT-87 & PMT-88 & PMT-98 & PMT-101 & \\
\hline Lithotype & & & & Basalt & Hawaiite & Basalt & Basalt & Hawaiite & Basalt & Hawaiite & Basalt & Basalt \\
\hline$w t . \%$ & & & & & & & & & & & & \\
\hline $\mathrm{SiO}_{2}$ & 47.15 & 46.76 & 47.03 & 48.14 & 50.00 & 47.35 & 48.09 & 48.67 & 47.49 & 47.51 & 47.44 & 47.68 \\
\hline $\mathrm{Al}_{2} \mathrm{O}_{3}$ & 13.17 & 13.55 & 13.79 & 14.33 & 15.26 & 14.46 & 14.46 & 14.36 & 13.89 & 14.26 & 14.12 & 14.77 \\
\hline $\mathrm{Fe}_{2} \mathrm{O}_{3}^{\mathrm{T}}$ & 10.54 & 10.19 & 10.29 & 12.04 & 12.19 & 12.10 & 12.27 & 13.85 & 13.48 & 13.82 & 11.83 & 10.89 \\
\hline $\mathrm{MnO}$ & 0.16 & 0.15 & 0.16 & 0.18 & 0.21 & 0.18 & 0.19 & 0.23 & 0.21 & 0.22 & 0.18 & 0.148 \\
\hline $\mathrm{MgO}$ & 11.74 & 11.65 & 11.20 & 6.93 & 4.67 & 6.63 & 6.52 & 4.41 & 6.21 & 5.18 & 8.51 & 8.64 \\
\hline $\mathrm{CaO}$ & 11.40 & 10.85 & 10.85 & 9.96 & 8.31 & 10.36 & 9.91 & 8.91 & 9.72 & 9.33 & 10.52 & 10.31 \\
\hline $\mathrm{Na}_{2} \mathrm{O}$ & 2.47 & 2.77 & 2.68 & 3.28 & 4.23 & 3.20 & 3.72 & 3.77 & 3.35 & 3.84 & 3.07 & 3.06 \\
\hline $\mathrm{K}_{2} \mathrm{O}$ & 0.87 & 0.84 & 0.90 & 1.17 & 1.39 & 1.21 & 1.06 & 1.38 & 1.25 & 1.42 & 0.86 & 0.71 \\
\hline $\mathrm{TiO}_{2}$ & 2.45 & 2.25 & 2.35 & 3.31 & 3.18 & 3.38 & 3.41 & 3.85 & 3.81 & 3.95 & 3.06 & 2.67 \\
\hline $\mathrm{P}_{2} \mathrm{O}_{5}$ & 0.38 & 0.35 & 0.40 & 0.73 & 1.30 & 0.78 & 0.84 & 1.41 & 1.14 & 1.46 & 0.83 & 0.34 \\
\hline LOI & -0.35 & -0.18 & -0.11 & -0.61 & -0.77 & -0.61 & -0.58 & -0.92 & -0.61 & -1.04 & -0.79 & -0.44 \\
\hline TOTAL & 99.98 & 99.19 & 99.54 & 99.44 & 99.96 & 99.03 & 99.89 & 99.92 & 99.94 & 99.94 & 99.62 & 98.80 \\
\hline Mg\# & 72.25 & 72.77 & 71.79 & 57.37 & 49.08 & 56.16 & 55.40 & 44.48 & 51.85 & 48.54 & 62.71 & 64.97 \\
\hline ppm & & & & & & & & & & & & \\
\hline Sc & 31 & 32 & 30 & 26 & 18 & 26 & 24 & 18 & 24 & 23 & 28 & 25 \\
\hline $\mathrm{Cu}$ & 79 & 83 & 75 & 44 & 22 & 40 & 41 & 15 & 37 & 24 & 47 & 33 \\
\hline $\mathrm{Ni}$ & 283 & 269 & 267 & 96 & 16 & 63 & 62 & $<1$ & 48 & 13 & 121 & 152 \\
\hline $\mathrm{Pb}$ & 2.6 & $<3$ & 1.7 & $<3$ & 2 & 2.8 & $<3$ & $<3$ & 2.4 & 1.7 & 1.1 & 2 \\
\hline $\mathrm{Zn}$ & $7 \overline{8}$ & 71 & $7 \overline{8}$ & 102 & $11 \overline{\overline{9}}$ & $8 \overline{9}$ & 95 & 98 & $9 \overline{5}$ & 80 & $8 \overline{1}$ & $8 \overline{1}$ \\
\hline V & 249 & 253 & 264 & 277 & 210 & 302 & 308 & 295 & 346 & 321 & 290 & 225 \\
\hline $\mathrm{Cr}$ & 706 & 668 & 617 & 230 & 23 & 189 & 194 & $<20$ & 161 & 46 & 310 & 314 \\
\hline Co & 48 & 49 & 48 & 36 & 23 & 36 & 37 & 29 & 37 & 31 & 43 & 47 \\
\hline $\mathrm{Ga}$ & 15 & 16 & 16 & 19 & 22 & 21 & 20 & 24 & 21 & 22 & 19 & 20 \\
\hline $\mathrm{Ge}$ & 1.3 & 1.3 & 1.4 & 1.4 & 1.5 & 1.3 & 1.2 & 1.4 & 1.5 & 1.6 & 1.2 & 1.6 \\
\hline $\mathrm{Rb}$ & 18 & 20 & 20 & 22 & 28 & 23 & 24 & 26 & 25 & 28 & 17 & 15 \\
\hline $\mathrm{Sr}$ & 445 & 420 & 430 & 523 & 678 & 560 & 531 & 697 & 538 & 565 & 542 & 464 \\
\hline $\mathrm{Y}$ & 21.1 & 21.3 & 22.2 & 31.2 & 46.3 & 35.5 & 35.4 & 51.7 & 40.1 & 46.2 & 31.3 & 27.1 \\
\hline $\mathrm{Zr}$ & 125 & 123 & 130 & 175 & 253 & 198 & 189 & 271 & 186 & 205 & 147 & 168 \\
\hline $\mathrm{Nb}$ & 30.1 & 29 & 29.4 & 38.4 & 56.5 & 43.5 & 49 & 67.2 & 51.2 & 53.6 & 39.4 & 35.1 \\
\hline Cs & 0.1 & 0.1 & 0.2 & $<0.1$ & 0.2 & 0.2 & $<0.1$ & 0.2 & $<0.1$ & 0.2 & $<0.1$ & 0.2 \\
\hline $\mathrm{Ba}$ & 289 & 286 & 309 & 458 & 883 & 474 & 468 & 414 & 607 & 746 & 441 & 188 \\
\hline La & 30 & 27.8 & 29.4 & 44.1 & 71.7 & 37.6 & 49.2 & 67.5 & 52.8 & 60.4 & 37.3 & 22.6 \\
\hline $\mathrm{Ce}$ & 59.7 & 54.9 & 57.7 & 89.3 & 145 & 77.4 & 99.9 & 140 & 109 & 125 & 77.4 & 48.1 \\
\hline $\mathrm{Pr}$ & 7.36 & 6.72 & 7.08 & 11.3 & 18.1 & 9.74 & 12.5 & 17.9 & 13.8 & 15.9 & 9.91 & 5.99 \\
\hline $\mathrm{Nd}$ & 30.9 & 28.1 & 29.7 & 48.1 & 76.7 & 42 & 52.4 & 76 & 58.8 & 67.9 & 43 & 26.4 \\
\hline $\mathrm{Sm}$ & 7.01 & 6.42 & 6.76 & 10.9 & 16.6 & 9.43 & 11.4 & 16.9 & 12.9 & 14.8 & 9.58 & 5.96 \\
\hline $\mathrm{Eu}$ & 2.5 & 2.21 & 2.27 & 3.9 & 6.04 & 3.39 & 4.09 & 5.89 & 4.73 & 5.53 & 3.82 & 2.22 \\
\hline Gd & 6.23 & 5.9 & 6.26 & 9.79 & 14.8 & 8.91 & 10.8 & 16.1 & 12.5 & 14.4 & 9.4 & 6.4 \\
\hline $\mathrm{Tb}$ & 1.01 & 0.98 & 1.02 & 1.54 & 2.23 & 1.37 & 1.62 & 2.37 & 1.8 & 2.04 & 1.37 & 1.02 \\
\hline Dy & 5.24 & 5.19 & 5.31 & 7.84 & 11.3 & 7.18 & 8 & 11.6 & 8.94 & 10.1 & 6.7 & 5.42 \\
\hline Ho & 0.98 & 0.98 & 1.02 & 1.44 & 2.05 & 1.34 & 1.48 & 2.1 & 1.64 & 1.85 & 1.24 & 0.92 \\
\hline $\mathrm{Er}$ & 2.4 & 2.48 & 2.58 & 3.55 & 5.01 & 3.35 & 3.69 & 5.09 & 4.01 & 4.47 & 3.06 & 2.46 \\
\hline $\mathrm{Tm}$ & 0.329 & 0.342 & 0.357 & 0.45 & 0.627 & 0.439 & 0.473 & 0.637 & 0.497 & 0.552 & 0.379 & 0.343 \\
\hline $\mathrm{Yb}$ & 1.87 & 1.98 & 2.06 & 2.71 & 3.68 & 2.62 & 2.77 & 3.77 & 2.99 & 3.31 & 2.24 & 2.07 \\
\hline $\mathrm{Lu}$ & 0.251 & 0.266 & 0.284 & 0.36 & 0.497 & 0.367 & 0.366 & 0.481 & 0.395 & 0.425 & 0.293 & 0.273 \\
\hline $\mathrm{Hf}$ & 3.6 & 3.7 & 3.8 & 5.1 & 6.9 & 5.1 & 5 & 6.9 & 5 & 5.3 & 3.9 & 4.5 \\
\hline Ta & 2.03 & 1.92 & 2.12 & 2.74 & 4.17 & 2.91 & 2.95 & 4.15 & 3.11 & 3.4 & 2.34 & 2.03 \\
\hline La & 30 & 27.8 & 29.4 & 44.1 & 71.7 & 37.6 & 49.2 & 67.5 & 52.8 & 60.4 & 37.3 & 22.6 \\
\hline Th & 1.79 & 1.62 & 1.68 & 2.29 & 3.76 & 2.88 & 2.74 & 3.96 & 2.91 & 3.28 & 2.09 & 2.3 \\
\hline $\mathrm{U}$ & 0.52 & 0.49 & 0.49 & 0.67 & 1.27 & 0.87 & 0.83 & 1.34 & 0.96 & 1.04 & 0.68 & 0.82 \\
\hline Volc. syst. & Santa Bárl & & & & & & & & & & & \\
\hline Sample & PMT-46 & PMT-63 & PMT-66 & & & -71 & -72 & PMT-73 & PMT-74 & PMT-77 & PMT-78 & PMT-83 \\
\hline$\overline{\text { Lithotype }}$ & $\overline{\text { Hawaiite }}$ & $\overline{\text { Hawaiite }}$ & $\overline{\text { Hawaiite }}$ & $\mathrm{Ba}$ & & $\mathrm{B}$ & & $\overline{\text { Mugearite }}$ & $\overline{\text { Mugearite }}$ & $\overline{\text { Hawaiite }}$ & $\overline{\text { Hawaiite }}$ & $\overline{\text { Benmoreite }}$ \\
\hline$w t . \%$ & & & & & & & & & & & & \\
\hline $\mathrm{SiO}_{2}$ & 51.17 & 49.30 & 50.13 & & & & 01 & 51.12 & 50.94 & 50.13 & 50.31 & 59.56 \\
\hline $\mathrm{Al}_{2} \mathrm{O}_{3}$ & 15.40 & 14.87 & 15.27 & & & & .42 & 15.86 & 16.04 & 16.83 & 16.87 & 16.77 \\
\hline $\mathrm{Fe}_{2} \mathrm{O}_{3}^{\mathrm{T}}$ & 12.47 & 12.90 & 12.08 & & & & .78 & 11.09 & 10.76 & 10.68 & 10.68 & 7.43 \\
\hline $\mathrm{MnO}$ & 0.20 & 0.21 & 0.20 & & & 17 & .17 & 0.18 & 0.18 & 0.17 & 0.17 & 0.20 \\
\hline $\mathrm{MgO}$ & 3.78 & 3.93 & 3.65 & & & 64 & .54 & 3.55 & 3.45 & 3.58 & 3.60 & 1.66 \\
\hline $\mathrm{CaO}$ & 8.15 & 8.06 & 7.98 & & & 84 & 63 & 7.68 & 7.72 & 8.50 & 8.54 & 3.93 \\
\hline $\mathrm{Na}_{2} \mathrm{O}$ & 3.91 & 4.09 & 4.14 & & & 72 & 69 & 4.68 & 4.63 & 4.17 & 4.25 & 5.68 \\
\hline $\mathrm{K}_{2} \mathrm{O}$ & 1.68 & 1.48 & 1.72 & & & 22 & 18 & 1.76 & 1.72 & 1.33 & 1.38 & 2.97 \\
\hline $\mathrm{TiO}_{2}$ & 3.38 & 3.40 & 3.26 & & & 70 & 62 & 2.92 & 2.82 & 2.98 & 3.00 & 1.39 \\
\hline $\mathrm{P}_{2} \mathrm{O}_{5}$ & 0.79 & 0.89 & 0.85 & & & 58 & .58 & 0.79 & 0.78 & 0.75 & 0.74 & 0.43 \\
\hline LOI & -0.63 & -0.12 & -0.31 & & & & & -0.51 & -0.47 & -0.08 & -0.30 & -0.29 \\
\hline
\end{tabular}


Table 1 (continued)

\begin{tabular}{|c|c|c|c|c|c|c|c|c|c|c|c|}
\hline \multirow{2}{*}{$\frac{\text { Volc. syst. }}{\text { Sample }}$} & \multicolumn{11}{|c|}{ Santa Bárbara } \\
\hline & \multirow{2}{*}{$\frac{\overline{\text { PMT-46 }}}{\text { Hawaiite }}$} & \multirow{2}{*}{$\frac{\text { PMT-63 }}{\text { Hawaiite }}$} & \multirow{2}{*}{$\frac{\text { PMT-66 }}{\text { Hawaiite }}$} & \multirow{2}{*}{$\frac{\text { PMT-70 }}{\text { Basalt }}$} & \multirow{2}{*}{$\frac{\text { PMT-71 }}{\text { Basalt }}$} & \multirow{2}{*}{$\frac{\text { PMT-72 }}{\text { Basalt }}$} & \multirow{2}{*}{$\frac{\text { PMT-73 }}{\text { Mugearite }}$} & \multirow{2}{*}{$\frac{\text { PMT-74 }}{\text { Mugearite }}$} & \multirow{2}{*}{$\frac{\text { PMT-77 }}{\text { Hawaiite }}$} & \multirow{2}{*}{$\frac{\text { PMT-78 }}{\text { Hawaiite }}$} & \multirow{2}{*}{$\frac{\text { PMT-83 }}{\text { Benmoreite }}$} \\
\hline Lithotype & & & & & & & & & & & \\
\hline \multicolumn{12}{|l|}{$w t . \%$} \\
\hline TOTAL & 100.30 & 99.01 & 98.96 & 99.84 & 100.17 & 100.29 & 99.11 & 98.57 & 99.04 & 99.24 & 99.74 \\
\hline Mg\# & 43.27 & 43.39 & 43.19 & 47.49 & 47.90 & 47.40 & 45.47 & 45.51 & 45.75 & 45.89 & 37.58 \\
\hline \multicolumn{12}{|l|}{ ppm } \\
\hline Sc & 17 & 17 & 17 & 18 & 19 & 19 & 16 & 14 & 16 & 15 & 8 \\
\hline $\mathrm{Cu}$ & 22 & 19 & 28 & 40 & 26 & 30 & 25 & 24 & 25 & 25 & 11 \\
\hline $\mathrm{Ni}$ & 3 & $<1$ & 6 & 34 & 33 & 30 & 5 & 7 & 7 & 8 & $<1$ \\
\hline $\mathrm{Pb}$ & 3.1 & $<3$ & $<3$ & 6 & 2.4 & 6.9 & $<3$ & 3.3 & 6 & $<3$ & $<3$ \\
\hline Zn & $12 \overline{2}$ & 124 & 113 & 97 & $9 \overline{2}$ & $9 \overline{3}$ & 114 & $10 \overline{8}$ & 108 & 110 & 121 \\
\hline V & 270 & 254 & 264 & 304 & 325 & 323 & 250 & 216 & 259 & 264 & 45 \\
\hline $\mathrm{Cr}$ & $<20$ & $<20$ & $<20$ & 48 & 62 & 61 & $<20$ & $<20$ & $<20$ & $<20$ & $<20$ \\
\hline Co & 27 & 24 & 24 & 34 & 35 & 35 & 24 & 22 & 24 & 25 & 5 \\
\hline Ga & 25 & 24 & 25 & 24 & 23 & 23 & 25 & 22 & 24 & 24 & 27 \\
\hline $\mathrm{Ge}$ & 1.4 & 1.4 & 1.4 & 1.4 & 1.3 & 1.4 & 1.4 & 1.4 & 1.4 & 1.5 & 1.6 \\
\hline $\mathrm{Rb}$ & 35 & 23 & 35 & 25 & 24 & 25 & 38 & 35 & 24 & 25 & 56 \\
\hline $\mathrm{Sr}$ & 607 & 634 & 647 & 717 & 695 & 685 & 648 & 599 & 686 & 694 & 498 \\
\hline $\mathrm{Y}$ & 44.3 & 43.7 & 47.6 & 34 & 32.7 & 32.7 & 45 & 40.1 & 39.7 & 39.6 & 53.7 \\
\hline $\mathrm{Zr}$ & 331 & 313 & 357 & 262 & 255 & 254 & 339 & 303 & 292 & 291 & 499 \\
\hline $\mathrm{Nb}$ & 69.6 & 63.2 & 73 & 57 & 57.6 & 57.1 & 73.1 & 67.1 & 65.4 & 64.4 & 100.5 \\
\hline Cs & 0.2 & 0.1 & 0.2 & 0.1 & $<0.1$ & $<0.1$ & $<0.1$ & $<0.1$ & 0.2 & 0.1 & $<0.1$ \\
\hline $\mathrm{Ba}$ & 457 & 395 & 445 & 331 & 306 & 301 & 424 & 379 & 353 & 359 & 627 \\
\hline La & 73.4 & 52.5 & 61.1 & 42.6 & 47.1 & 48.1 & 68 & 62.5 & 63.1 & 62.7 & 103.8 \\
\hline $\mathrm{Ce}$ & 144 & 107 & 120 & 84.2 & 92.9 & 95.5 & 134 & 122 & 123 & 123 & 187 \\
\hline $\mathrm{Pr}$ & 17.2 & 13.1 & 14.5 & 10.3 & 11.3 & 11.6 & 16.1 & 14.6 & 14.9 & 14.7 & 22.2 \\
\hline $\mathrm{Nd}$ & 69.6 & 54.7 & 60 & 42.5 & 45.6 & 47.2 & 63.7 & 58.1 & 59.3 & 59 & 82.5 \\
\hline $\mathrm{Sm}$ & 15.2 & 12.4 & 13.1 & 9.48 & 9.99 & 10.2 & 13.7 & 12.4 & 12.7 & 12.6 & 16.6 \\
\hline $\mathrm{Eu}$ & 4.76 & 4.04 & 4.15 & 3.09 & 3.33 & 3.35 & 4.46 & 3.97 & 4.09 & 4.08 & 5.13 \\
\hline Gd & 13.1 & 11.4 & 12 & 8.54 & 9.29 & 9.39 & 12.8 & 11.4 & 11.6 & 11.6 & 14.7 \\
\hline $\mathrm{Tb}$ & 2.11 & 1.76 & 1.89 & 1.35 & 1.4 & 1.41 & 1.94 & 1.72 & 1.78 & 1.76 & 2.31 \\
\hline Dy & 10.7 & 9.21 & 9.8 & 7.01 & 7.01 & 7.09 & 9.88 & 8.83 & 8.95 & 8.83 & 11.9 \\
\hline Ho & 1.95 & 1.72 & 1.81 & 1.29 & 1.31 & 1.3 & 1.81 & 1.64 & 1.67 & 1.63 & 2.28 \\
\hline $\mathrm{Er}$ & 4.89 & 4.24 & 4.61 & 3.29 & 3.19 & 3.29 & 4.56 & 4.17 & 4.18 & 4.08 & 5.84 \\
\hline $\mathrm{Tm}$ & 0.65 & 0.579 & 0.628 & 0.443 & 0.425 & 0.428 & 0.619 & 0.545 & 0.551 & 0.537 & 0.813 \\
\hline $\mathrm{Yb}$ & 3.8 & 3.38 & 3.69 & 2.56 & 2.54 & 2.61 & 3.66 & 3.29 & 3.26 & 3.22 & 4.79 \\
\hline Lu & 0.504 & 0.455 & 0.513 & 0.356 & 0.332 & 0.339 & 0.479 & 0.432 & 0.432 & 0.425 & 0.65 \\
\hline $\mathrm{Hf}$ & 8.9 & 8.1 & 8.9 & 6.6 & 6.2 & 6.3 & 8.4 & 7.5 & 7.4 & 7.5 & 12.2 \\
\hline $\mathrm{Ta}$ & 4.8 & 4.46 & 4.82 & 3.6 & 3.58 & 3.67 & 4.68 & 4.18 & 4.02 & 4.01 & 6.25 \\
\hline La & 73.4 & 52.5 & 61.1 & 42.6 & 47.1 & 48.1 & 68 & 62.5 & 63.1 & 62.7 & 103.8 \\
\hline Th & 4.89 & 5.26 & 5.88 & 3.94 & 3.89 & 3.98 & 5.72 & 5.14 & 4.58 & 4.53 & 8.53 \\
\hline $\mathrm{U}$ & 1.54 & 1.89 & 2.18 & 1.45 & 1.34 & 1.35 & 2.03 & 1.79 & 1.6 & 1.51 & 2.1 \\
\hline
\end{tabular}

westward increase in $\mathrm{Pb}$ isotopic ratios. The most unradiogenic sample was collected in the eastern part of the island (PMT-15 near Porto Judeu, see Fig. 1B for location), and more radiogenic values were obtained for the 1761 historic lava (PMT-27) and the Serreta sample, which are more similar to those values obtained for Santa Bárbara lavas (see insets of Fig. 6B and $\mathrm{C}$ ).

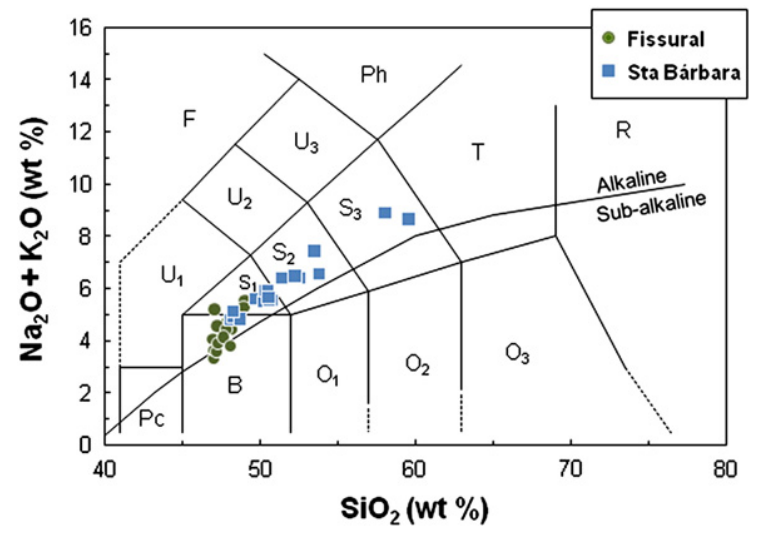

Fig. 3. Terceira lavas plotted on a TAS diagram (Le Bas et al., 1986). The black line represents the alkaline and sub-alkaline divider from Irvine and Baragar (1971). With the exception of sample PMT-65 (K-Trachybasalt), all samples plotted in $S_{1}, S_{2}$ and $S_{3}$ are classified as hawaiites, mugearites and benmoreites, respectively, given that $\left(\mathrm{Na}_{2} \mathrm{O}-2.0 \geq \mathrm{K}_{2} \mathrm{O}\right)$.
This work presents the first Hf isotopic data for Terceira Island. ${ }^{176} \mathrm{Hf} /{ }^{177} \mathrm{Hf}$ ratios range between 0.283002 and 0.283099 ( $\varepsilon \mathrm{Hf}:+8.1$ to +11.6$)$ with some samples displaying more depleted signatures than those reported for S. Miguel ( $\varepsilon \mathrm{Hf}:-0.2$ to +8.7$)$ and Pico ( $\varepsilon \mathrm{Hf}$ : +7.2 to +9.5 ) (see Beier et al., 2007; Elliott et al., 2007). Terceira samples tend to be less radiogenic than MORB lavas and plot slightly below the mantle array (Fig. 6D).

\section{Discussion}

Distinct elemental and isotopic signatures were presented for Fissural and Santa Bárbara lavas. However, before assessing the role of heterogeneities in the Terceira mantle source, it is important to evaluate the geochemical changes imprinted on primary magmas during their ascent to the surface.

\subsection{Fractional crystallization}

The distribution of mafic Terceira lavas in the TAS diagram (Fig. 3) allows for the recognition of two main groups: one formed by lavas from the Fissural volcanic system, plotting in the basaltic and hawaiitic compositional fields, and another formed by the lavas from the Santa Bárbara volcanic system, which display a much wider dispersion (from basalts to benmoreites). This compositional range is even larger when we consider the high volume of comenditic lava domes and coulées formed 


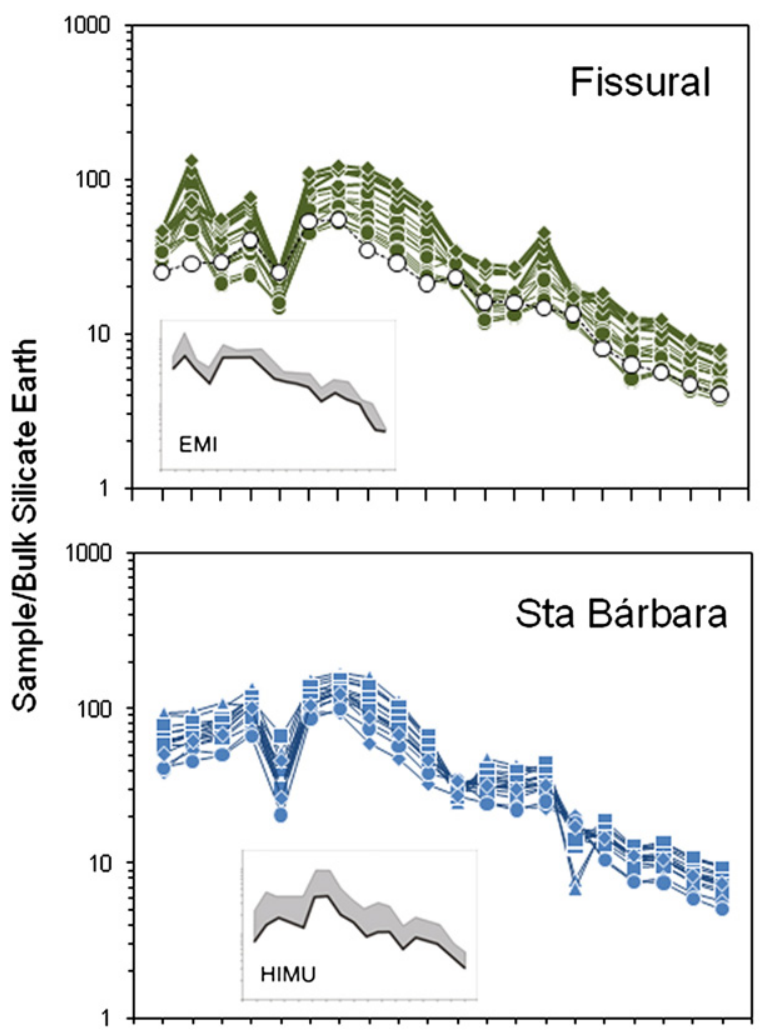

Rb Ba Th U K Nb Ta La Ce Nd Sr Zr Hif Sm Ti Dy Y Er Yo Lu
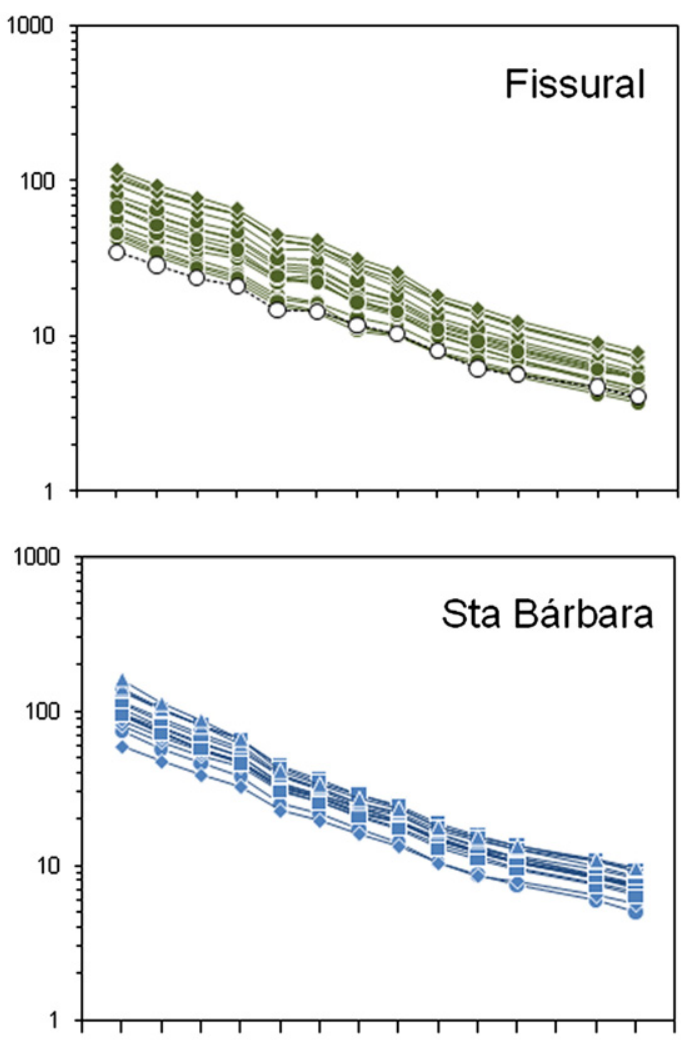

La Ce Pr Nd Sm Eu Gd Tb Dy Ho Er Tm Yo lu

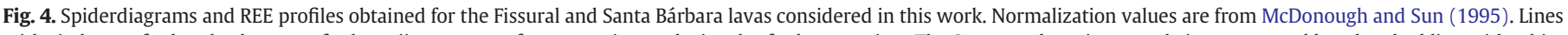

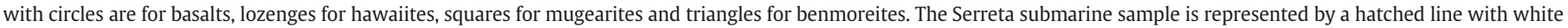
filled circles. Inset diagrams: typical patterns of lavas with strong HIMU and EM I signatures (from Weaver, 1991).

during the latest eruption events of the Santa Bárbara system (Self, 1976). This difference indicates the existence of a more mature magma chamber system feeding the Santa Bárbara volcano, which is also in accordance with the mixing of melt fractions at different evolutionary stages as interpreted from plagioclase phenocrysts (see Section 4.1.3). Despite the more primitive character shown by Fissural lavas, the presence of samples that can be considered primary magmatic liquids is scarce. Following the criteria of Frey et al. (1978), only the faintly porphyritic lavas sampled in the eastern area (PMT-8, PMT-14 e PMT-15, see Fig. 1B for location)

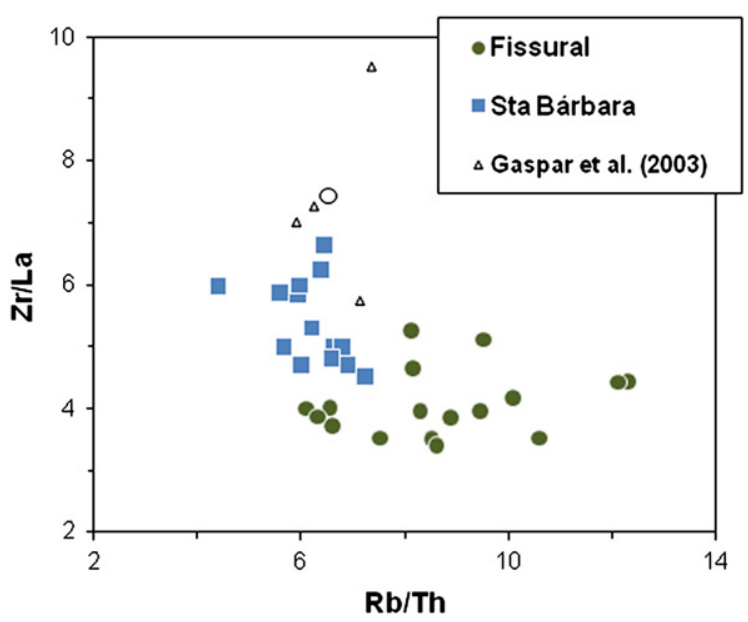

Fig. 5. Diagram ( $\mathrm{Zr} / \mathrm{La}$ vs. $\mathrm{Rb} / \mathrm{Th}$ ) showing the distinct elemental signatures of the Fissural and Santa Bárbara lavas. The Serreta submarine sample (white circle) erupted from the Fissural system is characterized by relatively high $\mathrm{Zr} / \mathrm{La}$ and low $\mathrm{Rb} / \mathrm{Th}$ ratios, more typical of the Santa Bárbara volcanic system. Data previously published for other Serreta lava balloons (Gaspar et al., 2003) are also presented. represent Terceira primary-like magma compositions (Mg\#: 71.8-72.8; Ni: 267-283 ppm). Their MgO contents (MgO: 11.34-11.83 wt.\%) are also in agreement with the proposal of Beier et al. (2008) for the composition of primary magmas erupted along the Terceira Rift ( $\mathrm{MgO} \sim 12.5 \mathrm{wt} . \%)$.

Based on distinct isotopic signatures and incompatible element ratios we demonstrated that the Fissural and Santa Bárbara volcanic systems are not comagmatic. Accordingly, we consider the apparent singe liquid line of descent shown in Fig. 7 as representing the evolution of a series of broadly similar, but not cogenetic, magma batches, evolving by similar processes in their travel to the surface.

Least-squares mass balance calculations considering pairs of less to more evolved magmas point to an amount of fractional crystallization between $50 \%$ and $65 \%$ to generate the observed range for each magmatic series. For the Fissural series, the lowest sum of squared residuals (0.246) was achieved by fractionating olivine ( 25.5\%), clinopyroxene $(\sim 53.7 \%)$, plagioclase $(\sim 14.8 \%)$ and $\mathrm{Cr}$-spinel $(\sim 6.0 \%)$. Plagioclase and titanomagnetite become dominant over olivine and $\mathrm{Cr}$-spinel when considering the most evolved samples. Concerning Santa Bárbara, the lowest sum of squared residuals (1.251) implies the fractionation of plagioclase $(\sim 42.9 \%)$, clinopyroxene $(\sim 40.4 \%)$, titanomagnetite $(\sim 15.4 \%)$ and minor olivine $(\sim 1.3 \%)$. The somewhat high value of the sum of squared residuals calculated for the Santa Bárbara series results from the large compositional range considered (from basalts to benmoreites) and from the onset of crystallization of accessory phases (e.g. apatite) during the late stages of magma evolution toward benmoreitic compositions.

The validity of these models can be tested by calculating the variation in the concentration of trace elements ( $\mathrm{Ni}, \mathrm{Sc}$ and $\mathrm{Sr}$ ) compatible with the fractionated mineral phases. Lines plotted in Fig. 8 (from A to C) were constructed considering the proportion of the minerals assessed from the mass balance calculations. These diagrams show a good fit between the model and the data. The same conclusion can be achieved from Fig. 8D, which emphasizes the role of the most abundant phenocrysts on major 
Table 2

Isotopic data determined for selected lavas erupted from the fissure zone (from PMT-8 to PMT-Serr-2) and from the Santa Bárbara volcano (from PMT-46 to PMT-74).

\begin{tabular}{|c|c|c|c|c|c|c|c|c|c|c|c|c|}
\hline Sample & ${ }^{87} \mathrm{Sr} /{ }^{86} \mathrm{Sr}$ & $2 \sigma$ & ${ }^{143} \mathrm{Nd} /{ }^{144} \mathrm{Nd}$ & $2 \sigma$ & ${ }^{176} \mathrm{Hf} /{ }^{177} \mathrm{Hf}$ & $2 \sigma$ & ${ }^{206} \mathrm{~Pb} /{ }^{204} \mathrm{~Pb}$ & $2 \sigma$ & ${ }^{207} \mathrm{~Pb} /{ }^{204} \mathrm{~Pb}$ & $2 \sigma$ & ${ }^{208} \mathrm{~Pb} /{ }^{204} \mathrm{~Pb}$ & $2 \sigma$ \\
\hline PMT-8 & 0.703472 & 0.000008 & 0.512923 & 0.000013 & 0.283043 & 0.000009 & - & - & - & - & - & - \\
\hline PMT-15 & 0.703523 & 0.000006 & 0.512908 & 0.000014 & 0.283002 & 0.000008 & 19.339 & 0.003 & 15.529 & 0.002 & 38.872 & 0.006 \\
\hline PMT-27 & 0.703550 & 0.000007 & 0.512954 & 0.000016 & 0.283073 & 0.000008 & 19.846 & 0.002 & 15.608 & 0.001 & 39.202 & 0.004 \\
\hline PMT-61 & 0.703455 & 0.000007 & 0.512948 & 0.000009 & 0.283074 & 0.000007 & 19.713 & 0.002 & 15.598 & 0.001 & 39.204 & 0.004 \\
\hline PMT-88 & 0.703425 & 0.000007 & 0.512963 & 0.000009 & 0.283076 & 0.000000 & 19.641 & 0.001 & 15.583 & 0.001 & 39.099 & 0.002 \\
\hline PMT-98 & 0.703433 & 0.000006 & 0.512962 & 0.000007 & 0.283069 & 0.000008 & 19.650 & 0.003 & 15.592 & 0.002 & 39.127 & 0.020 \\
\hline PMT-101 & 0.703458 & 0.000007 & 0.512961 & 0.000006 & 0.283078 & 0.000000 & 19.595 & 0.004 & 15.582 & 0.003 & 39.041 & 0.006 \\
\hline PMT-Serr-2 & 0.703596 & 0.000006 & 0.512980 & 0.000012 & 0.283099 & 0.000007 & 19.833 & 0.001 & 15.617 & 0.001 & 39.131 & 0.004 \\
\hline PMT-46 & 0.703557 & 0.000006 & 0.512961 & 0.000011 & 0.283072 & 0.000007 & 19.983 & 0.001 & 15.620 & 0.001 & 39.266 & 0.002 \\
\hline PMT-71 & 0.703571 & 0.000006 & 0.512965 & 0.000009 & 0.283061 & 0.000008 & 19.932 & 0.001 & 15.615 & 0.001 & 39.230 & 0.003 \\
\hline PMT-72 & 0.703572 & 0.000006 & 0.512969 & 0.000011 & 0.283072 & 0.000008 & 19.928 & 0.001 & 15.614 & 0.001 & 39.226 & 0.003 \\
\hline PMT-74 & 0.703573 & 0.000006 & 0.512956 & 0.000011 & 0.283084 & 0.000006 & 19.992 & 0.001 & 15.615 & 0.001 & 39.259 & 0.002 \\
\hline
\end{tabular}

element variation. The important role of clinopyroxene fractionation in both magmatic series is also expressed by the decrease of the $\mathrm{CaO} / \mathrm{Al}_{2} \mathrm{O}_{3}$ ratio along with magma evolution (Fig. 7). Moreover, the negative correlation between the $\mathrm{CaO} / \mathrm{Al}_{2} \mathrm{O}_{3}$ ratio and the alkalinity index [ $\left(\mathrm{Na}_{2} \mathrm{O}+\mathrm{K}_{2} \mathrm{O}\right)-0.37 *\left(\mathrm{SiO}_{2}-39\right)$; Albarède et al., 1997; not shown] demonstrates the influence of clinopyroxene fractionation on the increase of alkalinity of the Fissural basalts with differentiation. This is demonstrated by the $\left(\mathrm{Na}_{2} \mathrm{O}+\mathrm{K}_{2} \mathrm{O}\right) / \mathrm{SiO}_{2}$ ratio calculated for the pyroxene-dominated fractionating assemblage, which is significantly lower than that observed for the least evolved samples ( 0.014 versus 0.076, respectively). For the Santa Bárbara series, we note that the removal of Fe-Ti oxides can account for the compositional gap observed in the TAS diagram between mugearites and benmoreites (Fig. 3), given that their fractionation would trigger a sudden silica increase in the residual liquids (see also Mungall and Martin, 1995).

\subsection{Magma contamination}

The different incompatible trace element compositions of Fissural and Santa Bárbara magmas can be strengthened by comparing Santa Bárbara and Fissural lavas with similar MgO content (not shown; see Table 1). Santa Bárbara/Fissural normalized element ratios higher than unity were obtained for $\mathrm{Th}, \mathrm{U}, \mathrm{Zr}, \mathrm{Hf}$, and to a lesser extent $\mathrm{K}$ and $\mathrm{Rb}$, showing their enrichment in Santa Bárbara lavas when compared to
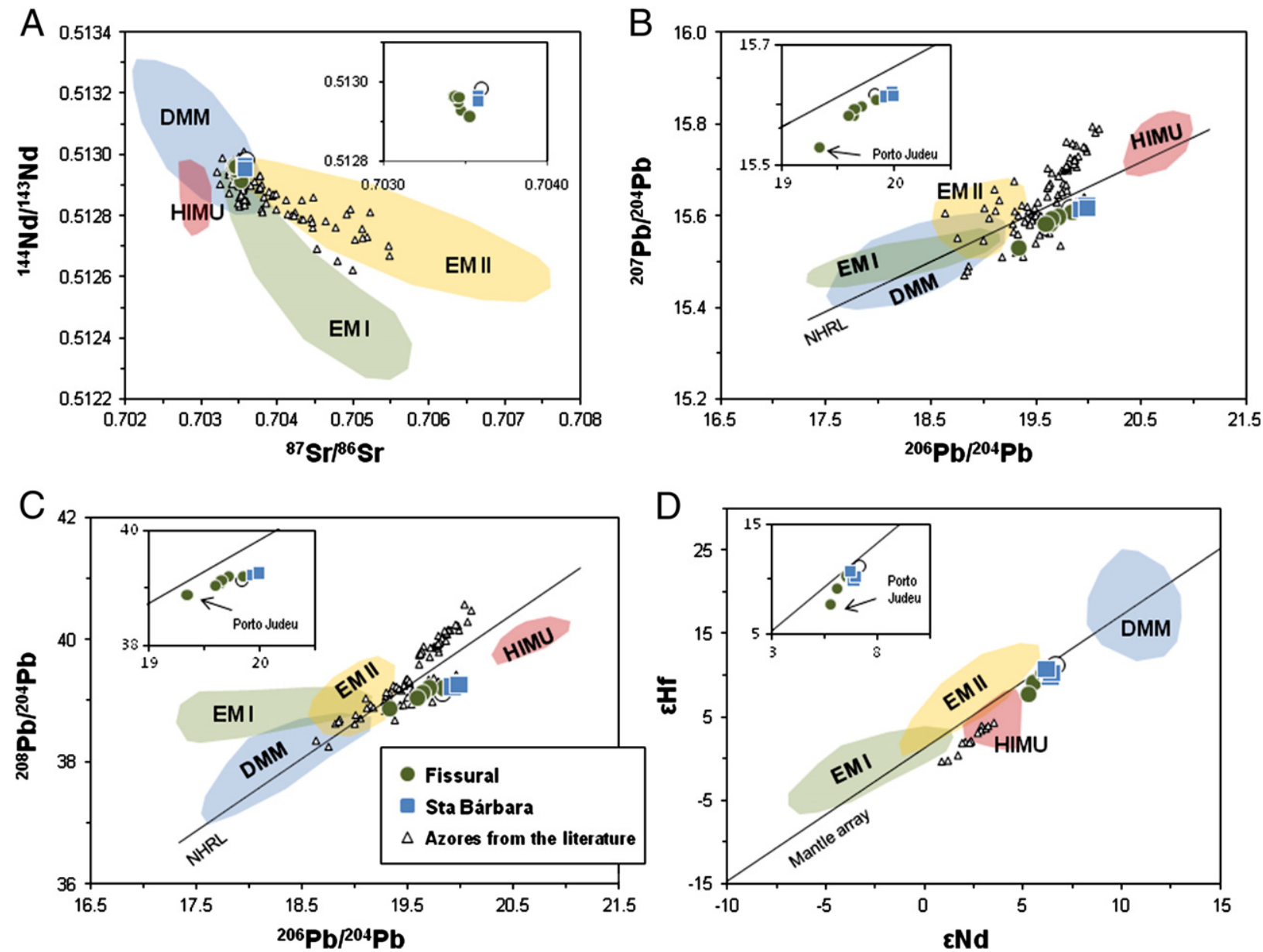

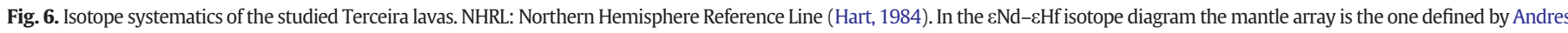

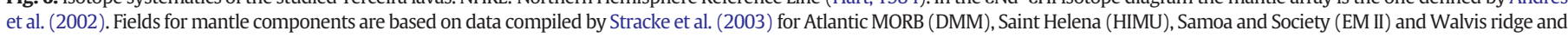
Pitcairn (EM I). The previously published Azores data is from the compilation made by Stracke et al. (2003), complemented with data from Elliott et al. (2007) and Beier et al. (2008). 

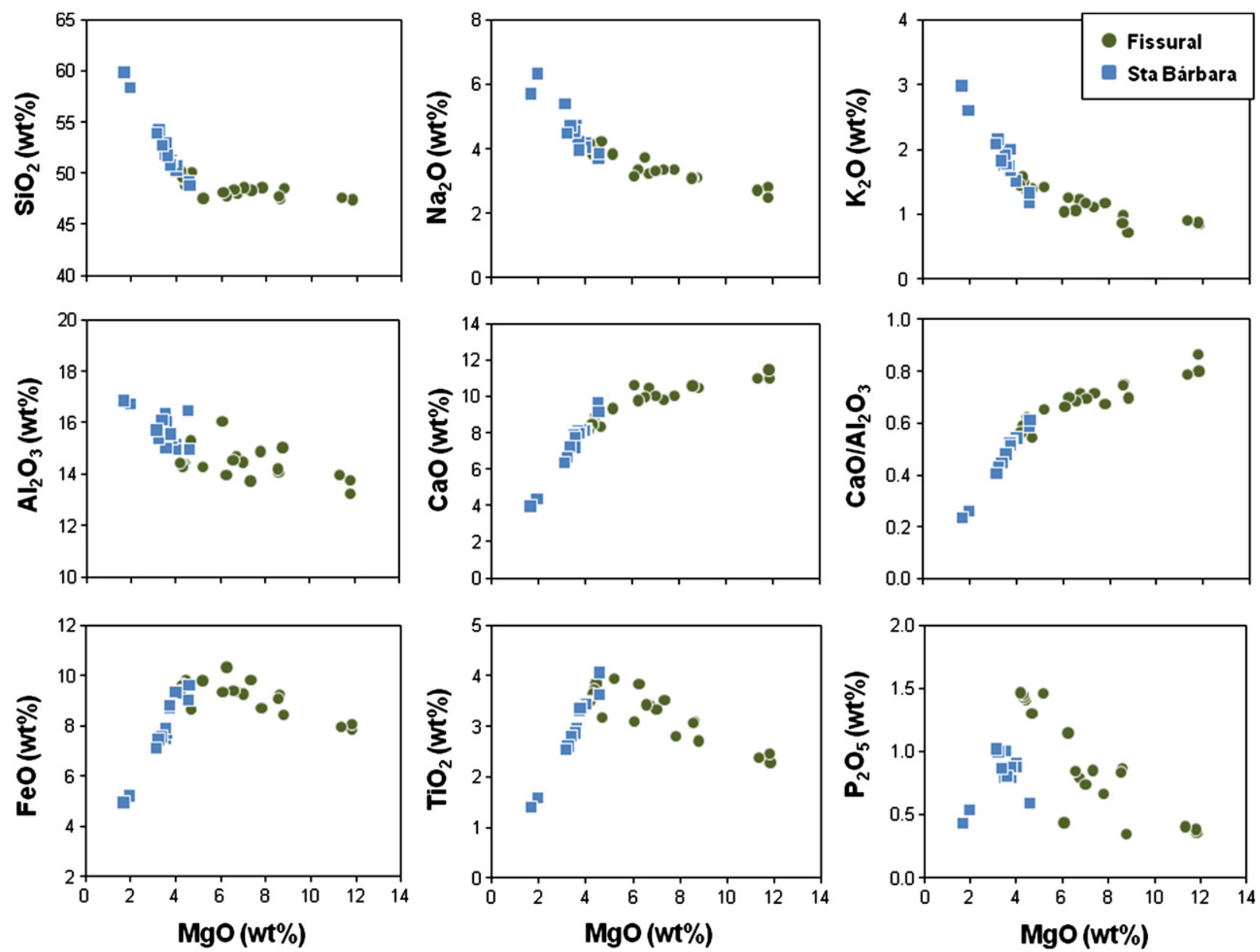

Fig. 7. Major element variation diagrams for Fissural and Santa Bárbara lavas.

those from the Fissural volcanic system. Conversely, calculated ratios for Ba and REE are lower than unity as the result of their relative depletion in Santa Bárbara magmas. These elements are not expected to be significantly fractionated by the mineral assemblages that were assumed to control the fractional crystallization process previously described. Thus, such differences can be related to source heterogeneity or, alternatively, to magma contamination. It is important to evaluate the latter possibility because most subaerial Fissural historical lavas were erupted in the vicinity of hyperalkaline trachytic lavas (pantellerites) belonging to the Pico Alto volcanic system (see Fig. 1B). However, trace element patterns of Fissural lavas reveal a lack of negative $\mathrm{Ba}, \mathrm{Sr}$ and Ti anomalies that would be expected as the result of the assimilation of those pantelleritic lavas (Fig. 9). Also, the absence of positive $\mathrm{Sr}$ and $\mathrm{Ti}$ anomalies precludes the assimilation of cumulates complementary to the pantellerites. Moreover there is no significant correlation $\left(\mathrm{R}^{2}<0.5\right)$ between the Ba anomaly and an evolution index ( $\mathrm{MgO}$ ) as would be expected from an assimilation and crystal fractionation $(\mathrm{ACF})$ process.

\subsection{Melting constraints}

At Terceira Island, primary magmas are only recognized for the Fissural volcanic system, which erupted lavas with $\mathrm{MgO} \sim 11$ to $12 \mathrm{wt}$. $\%$ in equilibrium with liquidus olivine (Fo90) at Porto Martins and Porto Judeu areas (see Fig. 1B for location). These olivines display low$\mathrm{NiO}$ contents $(<0.35 \mathrm{wt} . \%)$, precluding the possibility of a pyroxenitetype source for their host lavas (Sobolev et al., 2005).

Experimental work on the melting of dry peridotites (e.g., Hirose and Kushiro, 1993, 1998) has shown that $\mathrm{SiO}_{2}$ and $\mathrm{MgO}$ magma contents are independent of source composition, reflecting essentially the intensive parameters prevailing during magmatic extraction. Using the $\mathrm{MgO}$ and $\mathrm{SiO}_{2}$ contents of the primary magmas and the equations proposed by Albarèrede (1992), we estimate that magmatic extraction occurred at temperatures of $1350 \pm 40^{\circ} \mathrm{C}$ and pressures around $1.7 \pm 0.27 \mathrm{GPa}$.

The estimated temperature of magmatic segregation shows that Terceira primary magmas were hotter than the expected temperature of the asthenosphere $\left(\sim 1280^{\circ} \mathrm{C}\right.$, e.g., McKenzie and Bickle, 1988), which indicates a local temperature excess that is compatible with a thermally driven mantle plume (see also Putirka, 2008).

The depths estimated for magma extraction (from $\sim 50$ to $\sim 55 \mathrm{~km}$, considering an average crustal thickness of $12 \mathrm{~km}$, an oceanic crust density of $3.0 \mathrm{~g} / \mathrm{cm}^{3}$ and a mantle density of $3.3 \mathrm{~g} / \mathrm{cm}^{3}$ ) correspond to the spinel stability field, contrary to what would be anticipated from the REE fractionated patterns (Fig. 4; see also Fig. 10) or from the ${ }^{230} \mathrm{Th}$ excess over ${ }^{238} \mathrm{U}$ that were reported for Terceira lavas (Turner et al., 1997), both of which suggest melting in the presence of residual garnet. This contradiction has been described elsewhere and was explained by Eggins (1992) as the result of the onset of melting in the garnet stability zone (depths greater than $75 \mathrm{~km}$ ) and later re-equilibration and segregation at lesser depths within the spinel stability zone (see also Francis, 1995). A similar explanation was also given by Beier et al. (2008) for Terceira and Graciosa lavas. Moreover, these authors stressed that the age of the crust at the base of Terceira is compatible with a lithosphere thickness of $\sim 60 \mathrm{~km}$, which would support the migration of the melting front of a mantle diapir up to the spinel stability zone.

From the data distribution in the diagram of Fig. 10, a significant range of melting degrees of a garnet-bearing source is required to explain part of the trace element variability. This percept is 

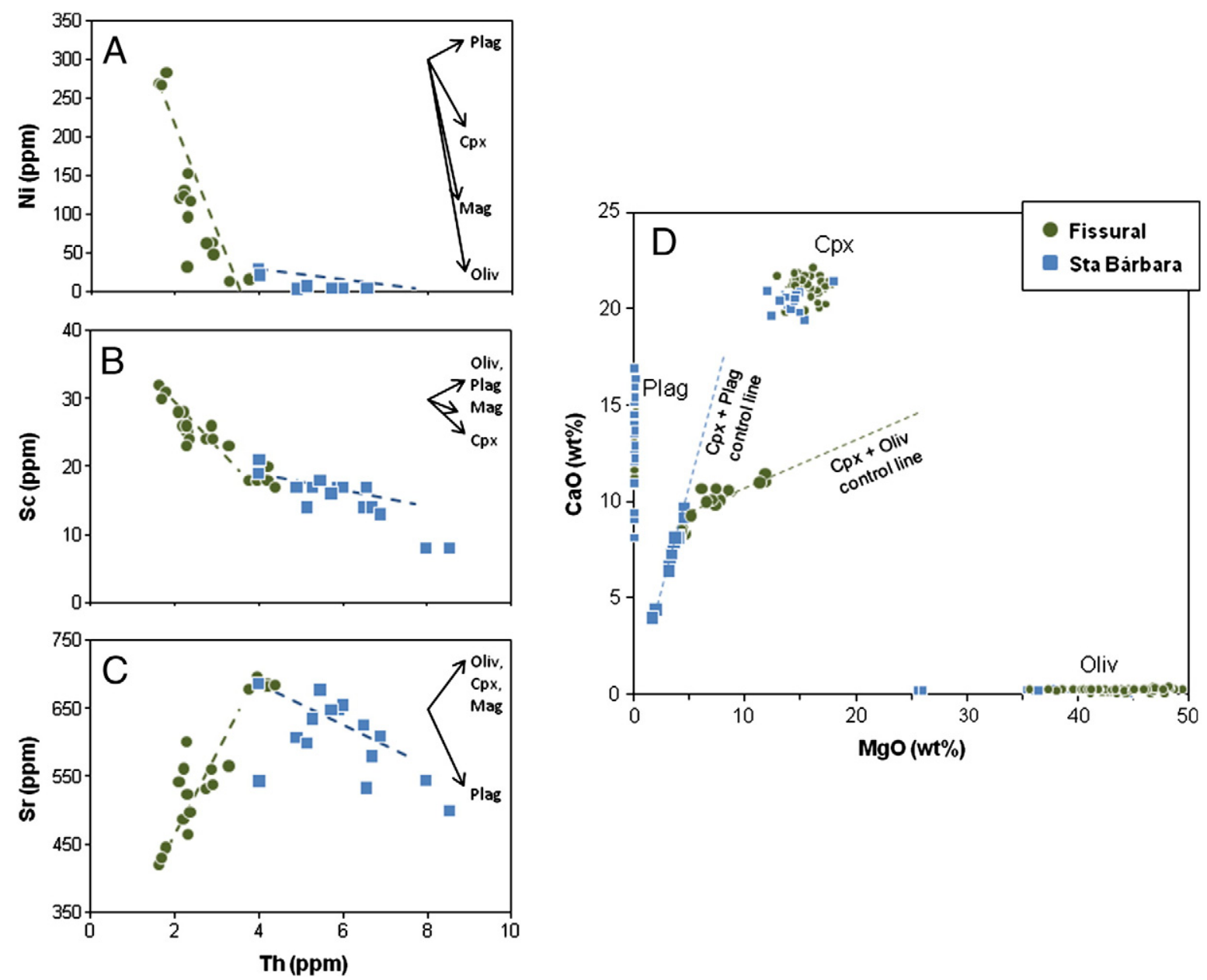

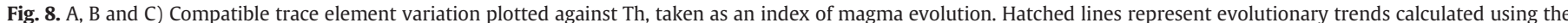

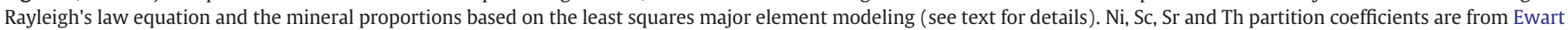

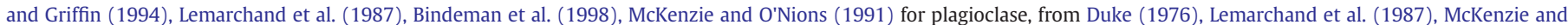

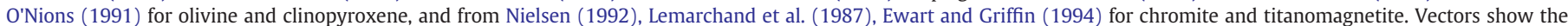

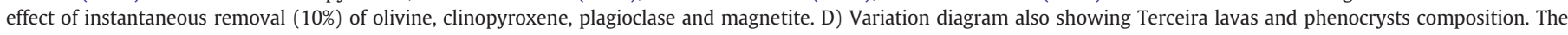

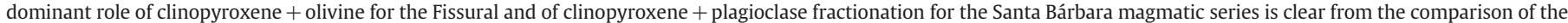
liquid lines of descent and the composition of the main phenocryst phases.

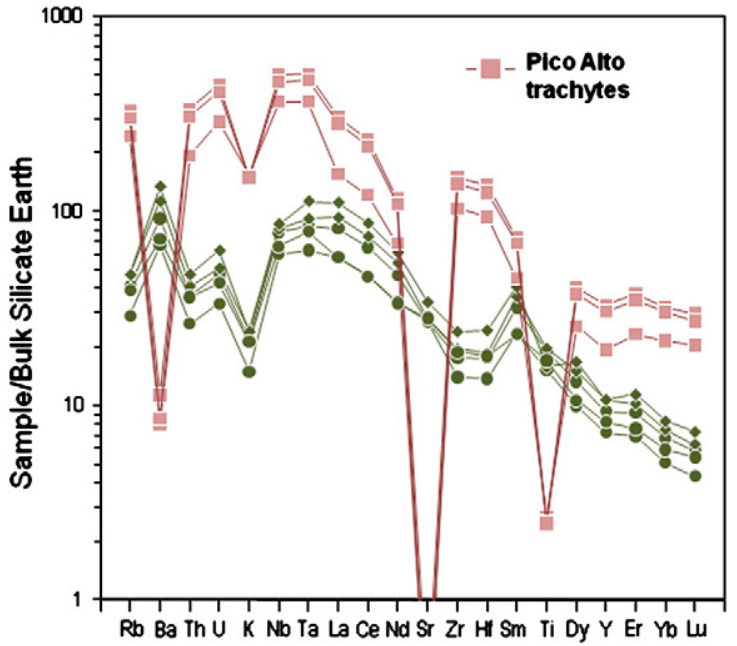

Fig. 9. Spiderdiagram comparing the Fissural lavas (that occur in the central Terceira Island) with the Pico Alto trachytes. Normalization values are from McDonough and Sun (1995). Fissural lavas are plotted with the same symbols as in Fig. 4.

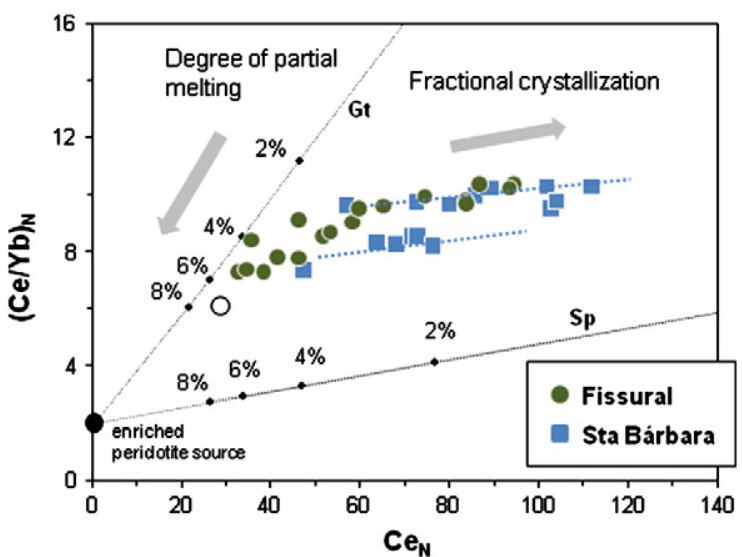

Fig. 10. Primitive mantle normalized $\mathrm{Ce} / \mathrm{Yb}$ ratios plotted against $(\mathrm{Ce})_{\mathrm{N}}$. Melting trends for garnet (Gt) and spinel (Sp) lherzolites were determined assuming an enriched mantle source with $4 \mathrm{ppm}$ and $0.5 \mathrm{ppm}$ of $\mathrm{Ce}$ and $\mathrm{Yb}$, respectively. The modeling was performed considering the batch melting of a source with $60 \% \mathrm{ol}+25 \% \mathrm{opx}+10 \% \mathrm{cpx}+5 \% \mathrm{gt}$ and $60 \%$ $\mathrm{ol}+25 \% \mathrm{opx}+10 \% \mathrm{cpx}+5 \% \mathrm{sp}$. The $\mathrm{Ce}$ and $\mathrm{Yb}$ partition coefficients were taken from McKenzie and O'Nions (1991). Note that the least evolved samples (lower Ce contents) plot close to the melting trend of the garnet lherzolite. White circle: Serreta sample. The dotted lines represent two sub-parallel trends formed by the Santa Bárbara samples, emphasizing the role of variable degrees of partial melting to the variability of the Terceira lavas. 
strengthened by the two sub-parallel trends formed by lavas from the same volcanic system (Santa Bárbara).

The higher degree of partial melting interpreted for the relatively primitive Serreta lava is interpreted as reflecting the presence of a thinner lithosphere according to the age decrease of the oceanic crust toward the MAR.

\subsection{Source implications}

As mentioned above, the significant differences in incompatible trace element ratios and isotopic signatures emerging from the comparison between the two studied volcanic systems (Fissural and Santa Bárbara) cannot be explained by fractional crystallization, assimilation and/or distinct degree of partial melting. From this perspective, these differences are believed to reflect the heterogeneity of the mantle domain(s) sampled by Terceira magmas.

In their study on Terceira Rift magmatism, Beier et al. (2008) showed the existence of significant heterogeneity at the scale of that structure, whereas smaller scale heterogeneity is evidenced by the existence of distinct intra-island magmatic signatures (e.g., Dupré et al., 1982; Haase and Beier, 2003; Millet et al., 2009). This study, which confirms the existence of mantle variability at the scale of a small island $\left(398 \mathrm{~km}^{2}\right)$, evidences that such heterogeneities are even detectable when two "coeval" volcanic systems erupted less than $10 \mathrm{~km}$ away.

The linear arrays observed in the ${ }^{207} \mathrm{~Pb} /{ }^{204} \mathrm{~Pb}$ versus ${ }^{206} \mathrm{~Pb} /{ }^{204} \mathrm{~Pb}$ space can be interpreted as secondary isochrones or, considering that the reference isotope is the same in both ratios, as binary mixing lines. Our data for the Fissural volcanic system define an array (see inset of Fig. 6) with a slope of $0.1621\left(R^{2}=0.965\right)$, which, if interpreted as an isochron, corresponds to an age of circa $2.47 \mathrm{Ga}$. Accordingly, this would be the age of the $\mathrm{U} / \mathrm{Pb}$ increase responsible for the positioning of the Terceira samples well to the right of the Geochron (e.g., Chase, 1981).

A linear array is also observed for the same magmatic series in the ${ }^{208} \mathrm{~Pb} /{ }^{204} \mathrm{~Pb}$ versus ${ }^{206} \mathrm{~Pb} /{ }^{204} \mathrm{~Pb}$ diagram, which can be used to decipher the meaning of both $\mathrm{Pb}$ linear arrays (e.g., Abouchami et al., 2000). If these arrays have an age meaning, a broad positive correlation should exist between the time-integrated $\mathrm{Th} / \mathrm{U}$ ratios inferred from $\mathrm{Pb}$ isotopic ratios ( $\mathrm{K}_{\mathrm{Pb}}$, Galer and O'Nions, 1985) and the elemental Th/ $\mathrm{U}$ measured in the samples. However, there is not a correlation between measured $\mathrm{Th} / \mathrm{U}$ ratio and $\mathrm{K}_{\mathrm{Pb}}$ (not shown) leading to the interpretation that the observed linear arrays represent the binary mixing of mantle components with different time-integrated compositions.

Santa Bárbara lavas plot close to the Terceira end-member defined by Beier et al. (2008) and clearly to the right of the Geochron, presenting ${ }^{206} \mathrm{~Pb} /{ }^{204} \mathrm{~Pb}$ ratios up to 19.992 (Fig. 11; Table 2). This implies that their sources were affected sometime in the past by a significant $\mathrm{U} / \mathrm{Pb}$ increase. They also depict trace element patterns (Fig. 4) similar to those characterizing typical HIMU-type basalts (Weaver, 1991). However, the ${ }^{206} \mathrm{~Pb} /{ }^{204} \mathrm{~Pb}$ ratios are significantly lower than those attributed to the more extreme HIMU signatures (e.g., Mangaia: 21.864; Woodhead, 1996). This implies the mixing of a high- $\mu$ end-member with a component characterized by significantly less radiogenic lead isotope signatures.

Millet et al. (2009) showed that the Azores lavas form two distinct trends on the $\Delta 8 / 4$ versus ${ }^{206} \mathrm{~Pb} /{ }^{204} \mathrm{~Pb}$ diagram, converging in the North Azores Fracture Zone MORB field (Fig. 12). This suggests that the unradiogenic- $\mathrm{Pb}$ component required by the isotopic signature of Santa Bárbara lavas is related to the mantle domain feeding the Mid-Atlantic Ridge. This is also endorsed by studies involving He and $\mathrm{Ne}$, which argue for mixing between a low ${ }^{21} \mathrm{Ne} /{ }^{22} \mathrm{Ne}$ and ${ }^{4} \mathrm{He} /{ }^{3} \mathrm{He}$ component that is required for Terceira Island and a radiogenic $\mathrm{He}$ and nucleogenic $\mathrm{Ne}$ end-member characterizing the unradiogenic- $\mathrm{Pb}$ component (Madureira et al., 2005; Moreira et al., 1999).

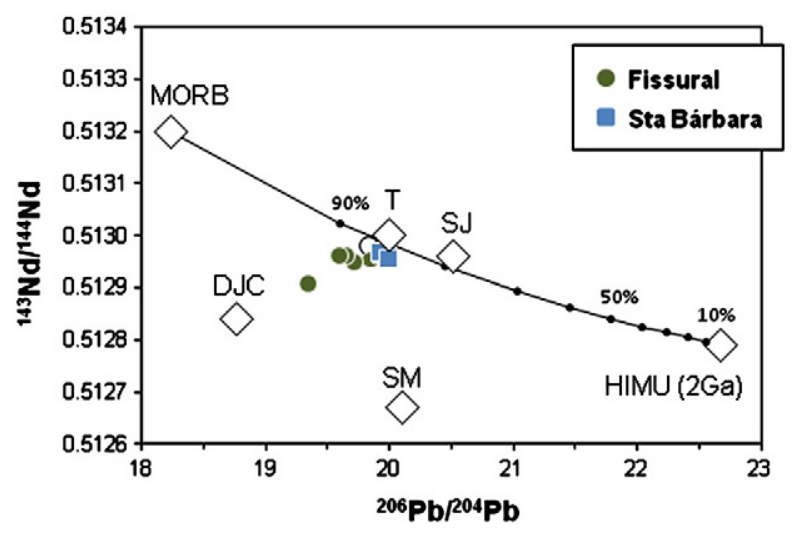

Fig. 11. Plot of Terceira lavas in the Pb-Nd isotopic space. Santa Bárbara and Serreta (white circle) lavas plot on the hypothetical mixing line between DMM and HIMU mantle components (Chauvel et al., 1992). The HIMU end-member is considered to represent ancient $(2.0 \mathrm{Ga}$ ) recycled oceanic crust. The latter was modeled by Chauvel et al. (1992) using a two-stage evolution model that considers $\mu_{1}=8.1$ and $\kappa_{1}=3.8$ (between $4.55 \mathrm{Ga}$ and $2 \mathrm{Ga}$ ) and $\mu_{2}=22$ and $\kappa_{2}=3.2$ (between $2 \mathrm{Ga}$ and present-day). Tick marks represent the DMM fraction within the mixture. T, SM, DJC: Terceira, S. Miguel and D. João de Castro Bank end-members (Beier et al., 2008); SJ: S. Jorge end-member (Millet et al., 2009). The trend defined by Fissural lavas is oblique to the DMM-HIMU mixing line, extending toward the less radiogenic D. João de Castro Bank end-member. Fissural lavas are also characterized by elemental ratios (e.g., Ba/Nb 10) clearly distinct from those resulting from the mixture between ancient recycled oceanic crust and DMM, both of which are typically characterized by $\mathrm{Ba} / \mathrm{Nb}<5$ (e.g., Weaver, 1991).

For Santa Bárbara, the proposed mixing between a HIMU-type end-member and a MORB-type one is supported by the ${ }^{143} \mathrm{Nd} /{ }^{144} \mathrm{Nd}$ versus ${ }^{206} \mathrm{~Pb} /{ }^{204} \mathrm{~Pb}$ diagram (Fig. 11). Conversely, Fissural lavas form a trend toward lower ${ }^{143} \mathrm{Nd} /{ }^{144} \mathrm{Nd},{ }^{206} \mathrm{~Pb} /{ }^{204} \mathrm{~Pb}$ and also ${ }^{176} \mathrm{Hf} /{ }^{177} \mathrm{Hf}$, ${ }^{207} \mathrm{~Pb} /{ }^{204} \mathrm{~Pb}$ and ${ }^{208} \mathrm{~Pb} /{ }^{204} \mathrm{~Pb}$ ratios (see Fig. 6; Table 2). These require the involvement of an end-member, characterized by a relatively lower time-integrated $\mathrm{Sm} / \mathrm{Nd}, \mathrm{Lu} / \mathrm{Hf}, \mathrm{U} / \mathrm{Pb}$ and $\mathrm{Th} / \mathrm{Pb}$ ratios as compared with the Terceira end-member sampled by Santa Bárbara lavas. Such more unradiogenic-Hf and -Nd end-member is, also, clearly distinct from the enriched end-member described at the eastern extremity of the Terceira Rift at S. Miguel Island (Fig. 11), for which several distinct origins have been proposed (cf. Beier et al., 2007; Elliott et al., 2007; Widom et al., 1997). Their isotopic signatures are more akin to those depicted by the end-member described by Beier et al. (2008) for D. João de Castro Bank (Fig. 11; Table 2), whose presence was not detected in Terceira Island by these authors.

The affinities of Fissural lavas with the D. João de Castro Bank endmember are reinforced by the relatively high $\mathrm{Ba} / \mathrm{Nb}$ ratios $(\sim 10)$

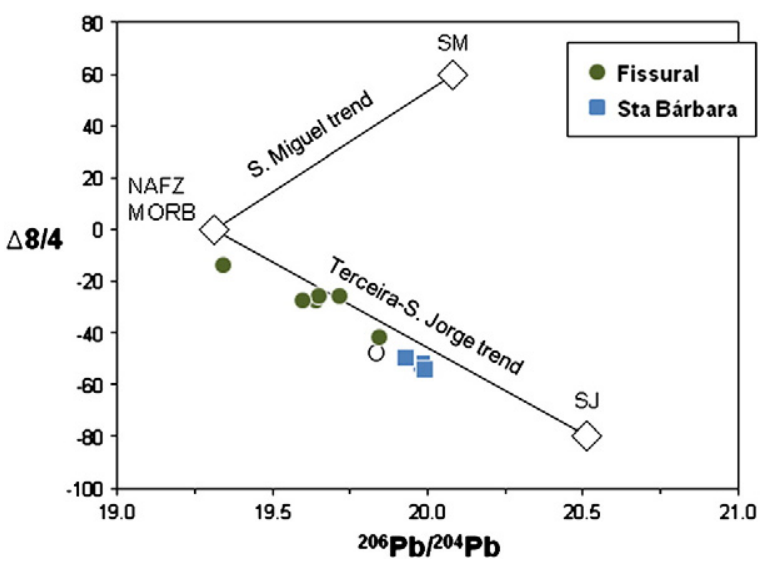

Fig. 12. Plot of Terceira lavas in the $\Delta 8 / 4$ versus ${ }^{206} \mathrm{~Pb} /{ }^{204} \mathrm{~Pb}$ diagram (following Millet et al., 2009). S. Miguel and Terceira-S. Jorge trends were obtained from correlations between average MORB from the MAR segment at the North Azores Fracture Zone (NAFZ MORB, Dosso et al., 1999) and S. Miguel and S. Jorge end-members (taken from Beier et al., 2008 and Millet et al., 2009). White circle: Serreta sample. 
characterizing lavas from the two volcanic systems (Table 1; Beier et al., 2008). The high $\mathrm{Ba} / \mathrm{Nb}$ ratios reflect either the mantle source composition or, alternatively, Ba-Nb fractionation during partial melting processes. These elements can only be fractionated in the presence of residual phlogopite, ilmenite or rutile (Bodinier et al., 1996; LaTourrette et al., 1995; Ryerson and Watson, 1987), contributing to decrease (phlogopite) or increase (ilmenite or rutile) of the $\mathrm{Ba} / \mathrm{Nb}$ ratio. However, residual ilmenite or rutile should be reflected by a negative Ti anomaly that is not observed in Fissural lavas. These also display $(\mathrm{Ce} / \mathrm{Nb})_{n}$ ratios ranging between 0.74 and 0.78 strongly arguing against $\mathrm{Nb}$ depletion. The latter, combined with the absence of negative $\mathrm{Ti}$ anomaly and high $\mathrm{Ba} / \mathrm{Nb}$ ratios point to the relative $\mathrm{Ba}$ enrichment in the mantle source (see also le Roex et al., 2010).

Fissural lavas are also characterized by $\mathrm{Nb} / \mathrm{U}$ ratios clearly higher (up to 60.4) than that of Santa Bárbara $(<45.5)$. Such characteristics of the Fissural lavas are not significantly distinct from those usually assigned to MORB and OIB ( $47 \pm 10$; Hofmann et al., 1986). However, we stress that $\mathrm{Nb} / \mathrm{U}$ is highly correlated with ${ }^{206} \mathrm{~Pb} /{ }^{204} \mathrm{~Pb}$ (Fig. 13), contrary to models invoking the interaction of plume magmas with a "recently" metasomatized amphibole-bearing oceanic lithosphere to explain relatively high $\mathrm{Nb} / \mathrm{U}$ ratios (e.g. Lundstrom et al., 2003; Millet et al., 2008). Considering the young age of the Terceira lithosphere $(<30 \mathrm{Ma})$, and that amphibole is not stable at the tempratures prevailing in the asthenosphere and plumes (Class and Goldstein, 1997), we suggest that the increase of $\mathrm{Nb} / \mathrm{U}$ ratio with the decrease of ${ }^{206} \mathrm{~Pb} /{ }^{204} \mathrm{~Pb}$, reflects the interaction of HIMU-type plume magmas with delaminated megaliths of sub-continental lithospheric mantle (SCLM) residing in the upper mantle beneath Terceira. The high $\mathrm{Nb} / \mathrm{U}$ of SCLM would have been imprinted by ancient metasomatic processes generating amphibole-rich domains (Lundstrom et al., 2003), which given amphibole/melt partition coefficients would also be characterized by low U/Pb ratios (e.g., LaTourrette et al., 1995).

Owing to their long-lived isolation from the convective asthenospheric mantle, SCLM is considered to be highly heterogeneous presenting compositions reflecting the age and tectonothermal events of the overlying crust (e.g., Griffin et al., 1999). However, there is growing evidence for the positioning of the SCLM below the Hf-Nd mantle array (e.g., Geldmacher et al., 2008; Griffin et al., 2000; Tappe et al., 2007) conferring plausibility to the hypothesis of a small contribution of SCLM to the Fissural magmas, as has been proposed based on other grounds, for the Azores (Millet et al., 2009; Moreira et al., 1999; O'Reilly et al., 2009; Widom and Shirey, 1996).

From the above discussion, we can conclude that the isotopic variability of the studied Terceira lavas reflects the mixing of several end-members. Santa Bárbara lavas reveal the mixing of an HIMU-like end-member that is believed to reflect the contribution of the mantle plume to the Terceira basalts, with the upper mantle domain feeding

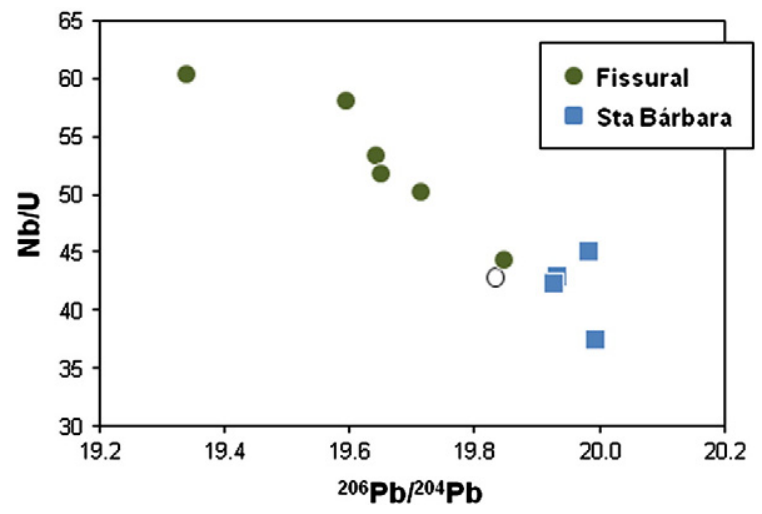

Fig. 13. Variation of the $\mathrm{Nb} / \mathrm{U}$ with the ${ }^{206} \mathrm{~Pb} /{ }^{204} \mathrm{~Pb}$ isotopic ratio. Note the negative correlation observed for the Fissural lavas. the Mid-Atlantic Ridge. Fissural lavas require an additional component with some characteristics that are similar to the SCLM.

HIMU signatures have been considered to stem from recycling and processing at the subduction zones of altered oceanic crust, resulting in the addition to the deep mantle of material characterized by low $\mathrm{Rb} /$ $\mathrm{Sr}$ and high $\mathrm{U} / \mathrm{Pb}$ ratios, which evolve over time to high ${ }^{206} \mathrm{~Pb} /{ }^{204} \mathrm{~Pb}$ and low ${ }^{87} \mathrm{Sr} /{ }^{86} \mathrm{Sr}$ ratios (e.g., Chauvel et al., 1992; Hofmann and White, 1982; Stracke et al., 2003; Weaver, 1991). These oceanic materials are believed to penetrate the Earth mantle as part of the downgoing slabs, which, as shown either by tomographic (e.g., Maruyama et al., 2007) or by density constraints (e.g., Hirose et al., 2005), can reach the lower levels of the mantle. The most Pb-radiogenic Santa Bárbara sample $\left({ }^{206} \mathrm{~Pb} /{ }^{204} \mathrm{~Pb}=19.992\right)$ presents a ${ }^{87} \mathrm{Sr} /{ }^{86} \mathrm{Sr}(0.70357)$ ratio slightly higher than the samples from HIMU type-localities, where this ratio is usually below 0.703 (e.g. Jackson and Dasgupta, 2008). For the Azores, this was also reported by Millet et al. (2009) in his study of S. Jorge Island suggesting that such relatively high ${ }^{87} \mathrm{Sr} /{ }^{86} \mathrm{Sr}$ ratios are characteristic of the HIMU-type end-member at the scale of the Azores archipelago.

At least a fourth contributor is required to explain the He and $\mathrm{Ne}$ isotopic characteristics described by Moreira et al. (1999) and Madureira et al. (2005) for Terceira Lavas. Indeed, the analyzed noble gases trapped in melt inclusions occurring in olivine phenocrysts $\left(\mathrm{R} / \mathrm{Ra}\right.$ up to $\left.11.4 ;{ }^{20} \mathrm{Ne} /{ }^{21} \mathrm{Ne}_{\text {corr }}=0.052\right)$ are clearly distinct from those assigned to mantle components similar to the dominant end-members in the Terceira sources (DMM as imaged by MORB: R/ $\mathrm{Ra}=8 \pm 1$, Zindler and Hart, $1986,{ }^{21} \mathrm{Ne} /{ }^{22} \mathrm{Ne}_{\mathrm{corr}} \sim 0.075$, Sarda et al., 2000; HIMU: R/Ra 5 to 7, Hanyu and Kaneoka, 1997; Moreira and Kurz, 2001; Parai et al., 2009, ${ }^{21} \mathrm{Ne} /{ }^{22} \mathrm{Ne}_{\text {corr }}=0.050$ to 0.066 , Parai et al., 2009). Those Terceira signatures, requiring the long term isolation of a low $(\mathrm{U}+\mathrm{Th}) /{ }^{3} \mathrm{He}$ and $(\mathrm{U}+\mathrm{Th}) /{ }^{20} \mathrm{Ne}$ reservoir (Class and Goldstein, 2005; Jackson et al., 2010; Porcelli and Elliott, 2008) have been considered to reflect the incorporation of lower mantle material in the Azores mantle domain (Madureira et al., 2005; Moreira et al., 1999). Therefore, there is a contradiction resulting from the apparent decoupling between the lithophile and $\mathrm{Pb}$ isotopic data, pointing to the existence of recycled materials, and $\mathrm{He}$ and Ne isotopic signatures, showing evidence for the contribution of lower mantle material. This has been explained elsewhere by assuming that the noble gas signatures of the deep mantle reservoir are virtually "unaffected" by the addition of crustal materials, which were almost completely stripped of He and Ne by degassing at mid-ocean ridges and, later, at subduction zones. In opposition, their lithophile, $\mathrm{Os}$ and $\mathrm{Pb}$ isotope signatures are strongly constrained by the recycled crustal material (e.g., Brandon et al., 2007; Jackson et al., 2009; Mata et al., 2010).

The heterogeneity of mantle plumes is thought to reflect either the role of entrainment of the surrounding mantle (e.g., Hauri et al., 1994) or the sampling of a heterogeneous thermal boundary layer (TBL) from which the plume rises (e.g., Farnetani et al., 2002). As shown above, the Azores mantle plume incorporates at least ancient recycled oceanic crust and a lower mantle contribution. Admitting that the extension associated with the Terceira Rift can only be regarded as a mechanism that facilitates the melting and/or magma extraction from the plume head imaged by seismic tomography (Yang et al., 2006) regional-scale studies can be used to infer information about the plume structure. Indeed, numerical simulations have shown that the spatial distribution of heterogeneities sampled by a volcanic province can provide insights on the plume and the TBL structures. A recent study of the Azores, also including the Flores and Corvo Islands located to the west of the MAR, suggest the existence of a chemical axisymmetric pattern, which, if reflecting a plume concentric zoning (Beier et al., 2010), would imply a layered TBL (see Farnetani and Hofmann, 2009, 2010).

Tomographic studies indicate that the Azores plume is centered NE of Terceira Island (Yang et al., 2006). However, a comparison of our results with those obtained by Millet et al. (2009) shows that S. Jorge 
lavas are significantly more $\mathrm{Pb}$-radiogenic $\left({ }^{206} \mathrm{~Pb} /{ }^{204} \mathrm{~Pb}\right.$ up to 20.5$)$ than those from Terceira $\left({ }^{206} \mathrm{~Pb} /{ }^{204} \mathrm{~Pb}\right.$ up to 19.992). This suggests that S. Jorge lavas carry to the surface a stronger plume signal than those at Terceira, where the HIMU signatures are more diluted by a stronger contribution of the local depleted upper mantle (see Fig. 11). We interpret this to reflect a larger degree of melting at Terceira as a result of the extensional regime characterizing the Terceira Rift.

\section{Concluding remarks}

Through the study of the volcanic systems (Santa Bárbara and Fissural) erupting mafic lavas at Terceira Island (Azores), we demonstrated the existence of present-day (less than $100 \mathrm{ka}$ ) small-scale lateral mantle source heterogeneity (Fig. 14). The western Terceira lavas, represented by the Santa Bárbara volcanic system and the Serreta ridge, sample the Terceira end-member defined by Beier et al. (2008) for the Terceira Rift. This end-member is here interpreted as the result of mixing between plume-derived ancient recycled oceanic crust (HIMU-type component) and the regional depleted MORB magmas/source. The D. João de Castro Bank end-member (Beier et al., 2008), not previously reported for Terceira Island, contributes to the Fissural volcanic system. It is suggested that the chemistry of these lavas reflects the interaction of the Azores plume with SCLM, the latter probably residing in the upper mantle as delaminated megaliths. Published noble gas data (Madureira et al., 2005; Moreira et al., 1999) on lavas from both volcanic systems also argue for the presence of a relatively undegassed component, which is interpreted to be inherited from the lower mantle reservoir sampled by the Azores plume.

The intra-volcanic system's chemical variability is mainly explained by variable proportions of the above-mentioned local end-members and by crystal fractionation processes. Magmatic evolution processes were more important for the Santa Bárbara volcanic system where magmas reach comenditic compositions. Fissural lavas range from basalts to hawaiites, with the latter occurring preferentially in the central part of the island where the crust is presumably thicker. As indicated by trace elements the partial mantle melting began in the garnet stability zone, although major element compositions suggest that magma segregation occurred within the spinel zone. A higher degree of partial melting was invoked for the Serreta submarine lava, which is interpreted to reflect the presence of a thinner lithosphere toward the MAR (Fig. 14).

Supplementary materials related to this article can be found online at doi:10.1016/j.lithos.2011.07.002.

\section{Acknowledgments}

This work was supported by the Fundação para a Ciência e a Tecnologia and Fundo Europeu de Desenvolvimento Regional through the EVOLV (PTDC/CTE-GIN/71838/2006-FCOMP-01-0124-FEDER-007129) and INOGAZ (PTDC/CTE-GIN/68851/2006) research projects. The authors thank M. Moreira, S. Martins and C. Mourão for fruitful discussions concerning the geochemistry of Terceira Island. We also acknowledge N. Lourenço, F. O. Marques, A. Hildenbrand, M. Miranda, J. Madeira, J. Luís and J. Carlos Nunes for the insights on the Terceira Island geology and the Azores evolution. Constructive comments and suggestions made by the Reviewers (A. le Roex, E. Widom, M. Millet) and also by the Editor (Nelson Eby), greatly contributed to the improvement of the manuscript.

\section{References}

Abouchami, W., Galer, S.J.G., Hofmann, A.W., 2000. High precision lead isotope systematics of lavas from the Hawaiian Scientific Drilling Project. Chemical Geology 169, 187-209. doi:10.1016/S0009-2541(00)00328-4.

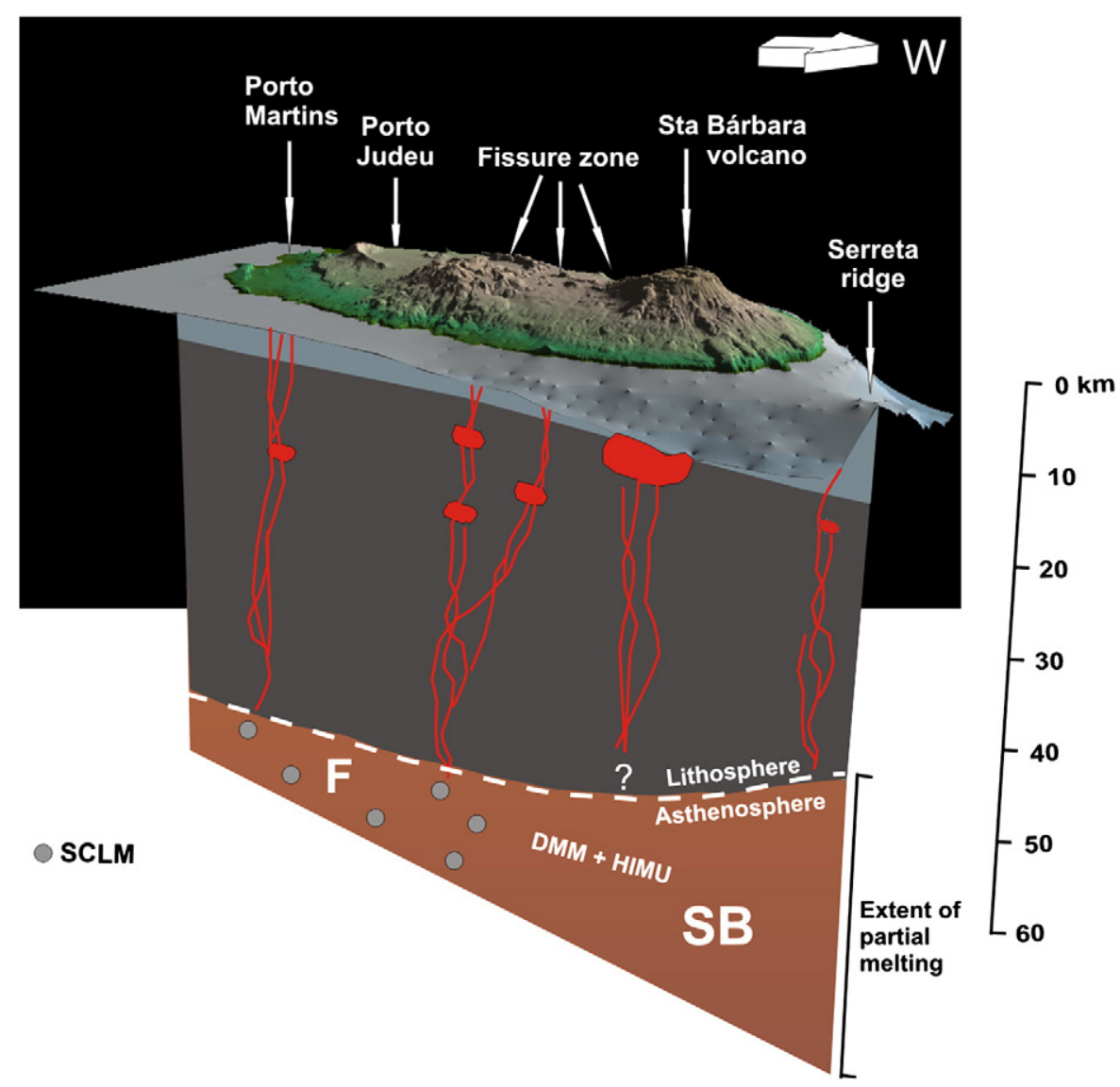

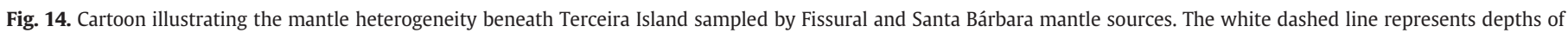
magma extraction, which is believed to be constrained by the thickness of the lithosphere. 
Albarède, F., Luais, B., Fitton, G., Semet, M., Kaminski, E., Upton, B., Bachèlery, P. Cheminée, J.-L., 1997. The Geochemical regimes of Piton de la Fournaise Volcano (Réunion) during the last 530000 years. Journal of Petrology 38 (2), 171-201.

Albarèrede, F., 1992. How deep do common basaltic magmas form and differentiate? Journal of Geophysical Research 97, 10997-11009.

Andres, M., Blichert-Toft, J., Schilling, J.-G., 2002. Hafnium isotopes in basalts from the southern Mid-Atlantic Ridge from $40^{\circ} \mathrm{S}$ to $55^{\circ} \mathrm{S}$ : discovery and Shona plume-ridge interactions and the role of recycled sediments. Earth and Planetary Science Letters 3 (10), 1-25. doi:10.1029/2002GC000324.

Beier, C., Stracke, A., Haase, K.M., 2007. The peculiar geochemical signatures of São Miguel lavas: metasomatised or recycled mantle sources? Earth and Planetary Science Letters 259 (1-2), 186-199. doi:10.1016/j.epsl.2007.04.038.

Beier, C., Haase, K.M., Abouchami, W., Krienitz, M.S., Hauff, F., 2008. Magma genesis by rifting of oceanic lithosphere above anomalous mantle: Terceira Rift, Azores. Geochemistry, Geophysics, Geosystems 9, Q12013. doi:10.1029/2008GC002112.

Beier, C., Turner, S., Plank, T., White, W., 2010. A preliminary assessment of the symmetry of source composition and melting dynamics across the Azores plume. Geochemistry, Geophysics, Geosystems 11 (2). doi:10.1029/2009GC002833.

Bindeman, I.N., Davis, A.M., Drake, M.J., 1998. Ion microprobe study of plagioclasebasalt partition experiments at natural concentration levels of trace elements. Geochimica et Cosmochimica Acta 62 (7), 1175-1193. doi:10.1016/S0016-7037 (98)00047-7.

Blichert-Toft, J., Chauvel, C., Albarède, F., 1997. Separation of Hf and Lu for highprecision isotope analysis of rocksamples by magnetic sector-multiple collector ICP-MS. Contributions to Mineralogy and Petrology 127, 248-260.

Bodinier, J.-L., Merlet, C., Bedini, R.M., Siemien, F., Remaidi, M., Garrido, C.J., 1996. Distribution of niobium, tantalum, and other highly incompatible trace elements in the lithospheric mantle: the spinel paradox. Geochimica et Cosmochimica Acta 60, 545-550.

Bonadiman, C., Beccaluva, L., Coltorti, M., Siena, F., 2005. Kimberlite-like metasomatism and "garnet signature" in spinel-peridotite xenoliths from Sal, Cape Verde archipelago: relics of a subcontinental mantle domain within the Atlantic Ocean lithosphere? Journal of Petrology 46, 2465-2493.

Bonatti, E., 1990. Not so "Hot Spots" in the oceanic mantle. Science 250, 107-110.

Brandon, A.D., Graham, D.W., Waight, T., Gautason, B., 2007. ${ }^{186}$ Os and ${ }^{187} \mathrm{Os}$ enrichments and high ${ }^{3} \mathrm{He} /{ }^{4} \mathrm{He}$ sources in the Earth's mantle: evidence from Icelandic picrites. Geochimica et Cosmochimica Acta 71, 4570-4591.

Brunelli, D., Seyler, M., 2010. Asthenospheric percolation of alkaline melts beneath the St. Paul region (Central Atlantic Ocean). Earth and Planetary Science Letters 289, 393-405.

Calvert, A., Moore, R., McGeehin, J., Rodrigues da Silva, A.M., 2006. Volcanic history and ${ }^{40} \mathrm{Ar} /{ }^{39} \mathrm{Ar}$ and ${ }^{14} \mathrm{C}$ geochronology of Terceira Island, Azores, Portugal. Journal of Volcanology and Geothermal Research 156 (1-2), 103-115. doi:10.1016/j. jvolgeores.2006.03.016.

Chaffey, D.J., Cliff, R.A., Wilson, B.M., 1989. Characterization of the St. Helena magma source. In: Saunders, A.D., Norry, M.J. (Eds.), Magmatism in the Ocean Basins: Geological Society Special Publication, 42, pp. 257-276.

Chase, C.G., 1981. Oceanic island Pb: two-stage histories and mantle evolution. Earth and Planetary Science Letters 52, 277-284.

Chauvel, C., Blichert-Toft, J., 2001. A hafnium isotope and trace element perspective on melting of the depleted mantle. Earth and Planetary Science Letters 190, 137-151.

Chauvel, C., Hofmann, A.W., Vidal, P., 1992. HIMU-EM: the French Polynesian connection. Earth and Planetary Science Letters 110, 99-119.

Class, C., Goldstein, S.L., 1997. Plume-lithosphere interaction in the ocean basins: constraints from the source mineralogy. Earth and Planetary Science Letters 150, $245-260$.

Class, C., Goldstein, S.L., 2005. Evolution of helium isotopes in the Earth's mantle. Nature 436, 1107-1112. doi:10.1038/nature03930.

Davies, G.R., Norry, M.J., Gerlach, D.C., Cliff, R.A., 1989. A combined chemical and Pb-Sr$\mathrm{Nd}$ isotope study of the Azores and Cape Verde hot-spots: the geodynamic implications. In: Saunders, A.D., Norry, M.J. (Eds.), Magmatism in the Ocean Basins: Geological Society Special Publication, 42, pp. 231-235.

Dosso, L., Bougault, H., Langmuir, C., Bonnier, O., Etoubleau, J., 1999. The age and distribution of mantle heterogeneity along the Mid-Atlantic Ridge $\left(31-41^{\circ} \mathrm{N}\right)$. Earth and Planetary Science Letters 170, 269-286.

Doucelance, R., Escrig, S., Moreira, M., Gariépy, C., Kurz, M., 2003. Pb-Sr-He isotope and trace element geochemistry of the Cape Verde Archipelago. Geochimica et Cosmochimica Acta 67, 3717-3733.

Duke, J.M., 1976. Distribution of the period four transition elements among olivine, calcic clinopyroxene and mafic silicate liquid; experimental results. Journal of Petrology 17 (4), 499-521.

Dupré, B., Allègre, C.J., 1983. Pb-Sr isotope variation in Indian Ocean basalts and mixing phenomena. Nature 303, 81-89.

Dupré, B., Lambret, B., Allégre, C.J., 1982. Isotopic variations within a single oceanic island: the Terceira case. Nature 299, 620-622.

Eggins, S.M., 1992. Petrogenesis of Hawaiian tholeiites: 2, aspects of dynamic melt segregation. Contributions to Mineralogy and Petrology 110, 398-410.

Elliott, T., Blichert-Toft, J., Heumann, A., Koetsier, G., Forjaz, V., 2007. The origin of enriched mantle beneath São Miguel, Azores. Geochimica et Cosmochimica Acta 71 (1), 219-240. doi:10.1016/j.gca.2006.07.043.

Ewart, A., Griffin, W.L., 1994. Application of proton-microprobe data to trace-element partitioning in volcanic-rocks. Chemical Geology 117 (1-4), 251-284. doi:10.1016 0009-2541(94)90131-7.

Farnetani, C.G., Hofmann, A.W., 2009. Dynamics and internal structure of a lower mantle plume conduit. Earth and Planetary Science Letters 282, 314-322.
Farnetani, C.G., Hofmann, A.W., 2010. Dynamics and internal structure of the Hawaiian plume. Earth and Planetary Science Letters 295, 231-240.

Farnetani, C.G., Legras, B.E., Tackley, P.J., 2002. Mixing and deformations in mantle plumes. Earth and Planetary Science Letters 196, 1-15.

Féraud, G., Kaneoka, I., Allègre, C.J., 1980. K/Ar ages and stress pattern in the Azores: geodynamic implications. Earth and Planetary Science Letters 46, 275-286.

Flower, M.F.J., Schmincke, H.-U., Bowman, H., 1976. Rare earth and other trace elements in historic Azorean lavas. Journal of Volcanology and Geothermal Research 1, 127-147.

Forjaz, V.H., Nunes, J.C., Bracéelos, P.J., 2004. Algar do Carvão-Ilha Terceira-Açores. In: Forjaz, V.H. (Ed.), Cartograma vulcanoambiental. Observatório Vulcanológico e Geotérmico, Açores.

França, Z.T., Tassinari, C.C., Cruz, J.V., Aparício, A.Y., Araña, V., Rodrigues, B.N., 2006. Petrology, geochemistry and $\mathrm{Sr}-\mathrm{Nd}-\mathrm{Pb}$ isotopes of the volcanic rocks from Pico Island-Azores (Portugal). Journal of Volcanology and Geothermal Research 156, 71-89.

Francis, D., 1995. The implications of picritic lavas for the mantle sources of terrestrial volcanism. Lithos 34, 89-105.

Frey, F.A., Green, D.H., Roy, S.D., 1978. Integrated models of basalt petrogenesis: a study of quartz tholeiites to olivine melilites from South Eastern Australia utilizing geochemical and experimental petrological data. Journal of Petrology 19, 463-513.

Galer, S.J.G., Abouchami, W., 1998. Practical application of lead triple spiking for correction of instrumental mass discrimination. Mineralogical Magazine 62A, 491-492.

Galer, S.J.G., O'Nions, R.K., 1985. Residence time of thorium, uranium and lead in the mantle with implications for mantle convection. Nature 316, 778-782.

Gaspar, J.L., Queiroz, G., Pacheco, J.M., Ferreira, T., Wallenstein, N., Almeida, M.H., Coutinho, R., 2003. Basaltic lava balloons producing during the 1998-2001 Serreta Submarine Ridge eruption (Azores). Geophysical Monograph 140, 205-212.

Gast, P.W., Tilton, G.R., Hedge, C., 1964. Isotopic composition of lead and strontium from Ascension and Gough Islands. Science 145, 1181-1185.

Geldmacher, J., Hoernle, K., Klügel, A., van den Bogaard, P., Bindeman, I., 2008. Geochemistry of a new enriched mantle type locality in the northern hemisphere: implications for the origin of the EM-I source. Earth and Planetary Science Letters 265, 167-182.

George, R., Rogers, N., 2002. Plume dynamics beneath the African plate inferred from the geochemistry of the Tertiary basalts of southern Ethiopia. Contributions to Mineralogy and Petrology 144, 286-304.

Gertisser, R., Self, S., Gaspar, J.L., Kelley, S.P., Pimentel, A., Eikenberg, J., Barry, T.L. Pacheco, J.M., Queiroz, G., Vespa, M., 2010. Ignimbrite stratigraphy and chronology on Terceira Island, Azores. Geological Society of America Special Paper 464, 133-154.

Griffin, W.L., O'Reilly, S.Y., Ryan, C.G., 1999. The composition and origin of subcontinental lithospheric mantle. In: Fei, Y., Bertka, C.M., Mysen, B.O. (Eds.), Mantle Petrology: Field Observations and High-pressure Experimentation: A Tribute to Francis R. (Joe) Boyd: Geochemical Society Special Publication, 6, pp. 13-45.

Griffin, W.L., Pearson, N.J., Belousova, E., Jackson, S.E., van Achterbergh, E., O'Reilly, S.Y., Shee, S.R., 2000. The Hf isotope composition of cratonic mantle: LAM-MC-ICPMS analysis of zircon megacrysts in kimberlites. Geochimica et Cosmochimica Acta 64 133-147.

Gurenko, A.A., Hoernle, K.A., Hauff, F., Schmincke, H.-U., Han, D., Miura, Y.N., Kaneoka, I., 2006. Major, trace element and $\mathrm{Nd}-\mathrm{Sr}-\mathrm{Pb}-\mathrm{O}-\mathrm{He}-\mathrm{Ar}$ isotope signatures of shield stage lavas from the central and western Canary Islands: insights into mantle and crustal processes. Chemical Geology 233, 75-112.

Haase, K.M., Beier, C., 2003. Tectonic control of ocean island basalt sources on São Miguel, Azores? Geophysical Research Letters 30 (16), 1856. doi:10.1029/ 2003GL017500.

Hanyu, T., Kaneoka, I., 1997. The uniform and low ${ }^{3} \mathrm{He} /{ }^{4} \mathrm{He}$ ratios of HIMU basalts as evidence for their origin as recycled materials. Nature 390, 273-276.

Hart, S.R., 1984. A large-scale isotope anomaly in the Southern Hemisphere mantle. Nature 309, 753-757.

Harte, B., 1987. Metasomatic events recorded in mantle xenoliths: an overview. In: Nixon, P.H. (Ed.), Mantle Xenoliths. John Willey \& Sons Ltd, pp. 625-640.

Hauri, E.H., Whitehead, J.A., Hart, S.R., 1994. Fluid dynamic and geochemical aspects of entrainment in mantle plumes. Journal of Geophysical Research 99 (B12) 24275-24300

Hildenbrand, A., Madureira, P., Marques, F.O., Cruz, I., Henry, B., Silva, P., 2008. Multistage evolution of a sub-aerial volcanic ridge over the last $1.3 \mathrm{Myr}$ : S. Jorge Island, Azores Triple Junction. Earth and Planetary Science Letters 273, 289-298.

Hildner, E., Klugel, A., Hauff, F., 2011. Magma storage and ascent during the 1995 eruption of Fogo, Cape Verde Archipelago. Contributions to Mineralogy and Petrology. doi:10.1007/s00410-011-0623-6.

Hirose, K., Kushiro, I., 1993. Partial melting of dry peridotites at high pressures; determination of compositions of melts segregated from peridotite using aggregates of diamond. Earth and Planetary Science Letters 114 (4), 477-489. doi:10.1016/0012-821X(93)90077-M.

Hirose, K., Kushiro, I., 1998. The effect of melt segregation on polybaric mantle melting: estimation from the incremental melting experiments. Physics of the Earth and Planetary Interiors 107 (1-3), 111-118.

Hirose, K., Takafuji, N., Sata, N., Ohishi, Y., 2005. Phase transition and density of subducted MORB crust in the lower mantle. Earth and Planetary Science Letters 237, 239-251.

Hirschmann, M.M., Ghiorso, M.S., 1994. Activities of nickel, cobalt, and manganese silicates in magmatic liquids and applications to olivine/liquid and to silicate/metal partitioning. Geochimica et Cosmochimica Acta 58, 4109-4126. 
Hofmann, A.W., 2003. Sampling mantle heterogeneity through oceanic basalts: Isotopes and trace elements. In: R.W. Carlson (Ed.), The Mantle and Core 2, pp.61-101. Treatise on Geochemistry (Eds. H. Holland and K.K. Turekian), ElsevierPergamon, Oxford.

Hofmann, A.W., White, W.M., 1982. Mantle plumes from ancient oceanic crust. Earth and Planetary Science Letters 57, 421-436.

Hofmann, A.W., Jochum, K.P., Seufert, M., White, W.M., 1986. Nb and Pb in oceanic basalts: new constraints on mantle evolution. Earth and Planetary Science Letters $79,33-45$.

Holm, P., Wilson, J., Christensen, B., 2006. Sampling the Cape Verde mantle plume: evolution of melt compositions on Santo Antão, Cape Verde Islands. Journal of Petrology 47, 145-189.

Irvine, T.N., Baragar, W.R.A., 1971. A guide to the chemical classification of the common volcanic rocks. Canadian Journal of Earth Science 8, 523-548.

Jackson, M.G., Dasgupta, R., 2008. Compositions of HIMU, EM1, and EM2 from global trends between radiogenic isotopes and major elements in ocean island basalts. Earth and Planetary Science Letters 276, 175-186.

Jackson, M.G., Kurz, M.D., Hart, S.R., 2009. Earth and Planetary Science Letters 287. 519-528.

Jackson, M.G., Carlson, R.W., Kurz, M.D., Kempton, P.D., Francis, D., Blusztajn, J., 2010. Evidence for the survival of the oldest terrestrial mantle reservoir. Nature 466 853-858. doi:10.1038/nature09287.

Jean-Baptiste, P., Allard, P., Coutinho, R., Ferreira, T., Fourré, E., Queiroz, G., Gaspar, J.L., 2009. Helium isotopes in hydrothermal volcanic fluids of the Azores archipelago. Earth and Planetary Science Letters 281, 70-80.

Jurewicz, A.J., Watson, E.B., 1988. Cations in olivine, Part 1: calcium partitioning and calcium magnesium distribution between olivines and coexisting melts, with petrologic applications. Contributions to Mineralogy and Petrology 99, 176-185.

LaTourrette, T., Hervig, R.L., Holloway, J.R., 1995. Trace element partitioning between amphibole, phlogopite, and basanite melt. Earth and Planetary Science Letters 135 $13-30$

Le Bas, M.J., Le Maiter, R.W., Strekeisen, A.L., Zanettin, B., 1986. A chemical classification of volcanic rocks based on the Total Alkali-Silica Diagram. Journal of Petrology 27, $745-750$.

le Roex, A., Class, C., O'Connor, J., Jokat, W., 2010. Shona and discovery Aseismic Ridge Systems, South Atlantic: trace element evidence for enriched mantle sources. Journal of Petrology 51, 2089-2120.

Lemarchand, F., Benoit, V., Calais, G., 1987. Trace element distribution coefficients in alkaline series. Geochimica et Cosmochimica 51, 1071-1081. doi:10.1016/0016 7037(87)90201-8.

Libourel, G., 1999. Systematics of calcium portioning between olivine and silicate melt: implications for melt structure and calcium content of magmatic olivines. Contributions to Mineralogy and Petrology 136, 63-80.

Lourenço, N., 2007. Tectono-magmatic processes at the Azores Triple Junction. PhD thesis. Universidade do Algarve, $239 \mathrm{pp}$.

Lourenço, N., Miranda, J.M., Luis, J.F., Ribeiro, A., Mendes-Victor, L., Madeira, J., Needham, H. 1998. Morpho-tectonic analysis of the Azores Volcanic Plateau from a new bathymetric compilation of the area. Marine Geophysical Research 20, 141-156.

Luís, J.F., Miranda, J.M., 2008. Reevaluation of magnetic chrons in the North Atlantic between $35^{\circ} \mathrm{N}$ and $47^{\circ} \mathrm{N}$ : implications for the formation of the Azores Triple Junction and associated plateau. Journal of Geophysical Research 113, B10105. doi:10.1029/2007/JB005573.

Lundstrom, C.C., Hoernle, K., Gill, J., 2003. U-series disequilibria in volcanic rocks from the Canary Islands: plume versus lithospheric melting. Geochimica et Cosmochimica Acta 67, 4153-4177.

Madeira, J., 2005. The volcanoes of Azores Islands: a worl-class heritage (examples from Terceira, Pico and Faial Islands). IV International Symposium ProGEO on the Conservation of the Geological Heritage, Field Trip Guide Book. 104 pp.

Madureira, P., Moreira, M., Mata, J., Allègre, C.J., 2005. Primitive helium and neon isotopes in Terceira Island: constraints on the origin of the Azores archipelago. Earth and Planetary Science Letters 233, 429-440.

Martins, S., Mata, J., Munhá, J., Mendes, M.H., Maerschalk, C., Caldeira, R., Mattielli, N., 2010. Chemical and mineralogical evidence of the occurrence of mantle metasomatism by carbonate-rich melts in an oceanic environment (Santiago Island, Cape Verde). Mineralogy and Petrology 99, 43-65.

Maruyama, S., Santos, M., Zhao, D., 2007. Superplume, supercontinent, and postperovskite: mantle dynamics and anti-plate tectonics on the Core-Mantle Boundary. Gondwana Research 11, 7-37.

Mata, J., Munhá, J., 2004. Madeira Island alkaline lava spinels: petrogenetic implications. Mineralogy and Petrology 81, 85-111.

Mata, J., Kerrich, R., MacRae, N.D., Wu, T.-U., 1998. Elemental and isotopic (Sr, Nd, and $\mathrm{Pb})$ characteristics of Madeira Island basalts: evidence for a composite HIMU-EM plume fertilizing lithosphere. Canadian Journal of Earth Science 35, 980-997.

Mata, J., Moreira, M., Doucelance, R., Ader, M., Silva, L.C., 2010. Noble gas and carbon isotopic signatures of Cape Verde oceanic carbonatites: implications for carbon provenance. Earth and Planetary Science Letters 291, 70-83.

Mattielli, N., Weis, D., Scoates, J.S., Shimizu, N., Mennessier, J.-P., Grégoire, M., Cottin, J.-Y. Giret, A., 1999. Evolution of heterogeneous lithospheric mantle in a plume environment beneath the Kerguelen Archipelago. Journal of Petrology 40, 1721-1744.

Mattielli, N., Weis, D., Blichert-Toft, J., Albarède, F., 2002. Hf isotope evidence for a Miocene change in the Kerguelen mantle plume composition. Journal of Petrology 43, 1327-1339.

McDonough, W.F., Sun, S.-S., 1995. The composition of the earth. Chemical Geology 120 223-253.

McKenzie, D., Bickle, M.J., 1988. The volume and composition of melt generated by extension of the lithosphere. Journal of Petrology 29, 625-679.
McKenzie, D., O'Nions, R.K., 1991. Partial melt distributions from inversion of rare earth element concentrations. Journal of Petrology 32, 1021-1091.

Middlemost, E.A., 1989. Iron oxidation ratios, norms and the classification of volcanic rocks. Chemical Geology 77, 19-26.

Millet, M.-A., Doucelance, R., Schiano, P., David, K., Bosq, C., 2008. Mantle plume heterogeneity versus shallow-level interactions: a case study, the São Nicolau Island, Cape Verde archipelago. Journal of Volcanology and Geothermal Research 176, 265-276.

Millet, M.-A., Doucelance, R., Baker, J.A., Schiano, P., 2009. Reconsidering the origins of isotopic variations in Ocean Island Basalts: insights from fine-scale study of São Jorge Island, Azores archipelago. Chemical Geology 265, 289-302.

Miranda, J., Victor, L.M., Simões, J., Luis, J., Matias, L., Shimamura, H., Shiobara, H., Nemoto, H., Mochizuki, H., Hirn, A., Lépine, J., 1998. Tectonic setting of the Azores Plateau deduced from a OBS survey. Marine Geophysical Research 20, 171-182.

Moreira, M. Kurz, M.D., 2001. Subducted oceanic lithosphere and the origin of the 'high $\mu$ ' basalt helium isotopic signature. Earth and Planetary Science Letters 189, 49-57.

Moreira, M., Doucelance, R., Dupré, B., Kurz, M., Allègre, C.J., 1999. Helium and lead isotope geochemistry in the Azores archipelago. Earth and Planetary Science Letters 169, 189-205.

Morimoto, N., Fabries, J., Ferguson, A.K., Ginzburg, I.V., Ross, M., Seifert, F.A., Zussman, J., Aoki, K., Gottardi, G., 1988. Nomenclature of pyroxenes. American Mineralogist 73, 1123-1133.

Mungall, J.E., Martin, R.F., 1995. Petrogenesis of basalt-comendite and basaltpantellerite suites, Terceira, Azores, and some implications for the origin of ocean-island rhyolites. Contributions to Mineralogy and Petrology 119, 43-55

Nielsen, R.L., 1992. BIGD: a FORTRAN program to calculate trace-element partition coefficients for natural mafic and intermediate composition magmas. Computers and Geosciences 18, 773-788.

Nunes, J.C., 2000. Notas sobre a geologia da Ilha Terceira (Açores). Açoreana 9 (2), 205-215.

O'Reilly, S., Zhang, M., Griffin, W.L., Begg, G., Hronsky, J., 2009. Ultradeep continental roots and their oceanic remnants: a solution to the geochemical "mantle reservoir" problem? Lithos 211S, 1043-1054

Parai, R., Mukhopadhyay, S., Lassiter, J.C., 2009. New constraints on the HIMU mantle from neon and helium isotopic compositions of basalts from the Cook-Austral Islands. Earth and Planetary Science Letters 253, 253-261.

Porcelli, D., Elliott, T., 2008. The evolution of He Isotopes in the convecting mantle and the preservation of high ${ }^{3} \mathrm{He} /{ }^{4} \mathrm{He}$ ratios. Earth and Planetary Science Letters 269, 175-185.

Putirka, K., 2008. Excess temperatures at ocean islands: implications for mantle layering and convection. Geology 36 (4), 283-286.

Reisberg, L., Zindler, A., 1986. Extreme variations in the upper mantle: evidence from Ronda. Earth and Planetary Science Letters 81, 29-45.

Ryerson, F.J., Watson, E.B., 1987. Rutile saturation in magmas: implications for Ti-NbTa depletion in island-arc basalts. Earth and Planetary Science Letters 86, 225-239.

Sarda, P., Moreira, M., Staudacher, T., Schilling, J.-G., Allègre, C.J., 2000. Rare gas systematics on the southernmost Mid-Atlantic Ridge: constraints on the lower mantle and the Dupal source. Journal of Geophysical Research 105, 5973-5996.

Schminke, H.U., 1973. Magmatic evolution and tectonic regime in the Canary, Madeira and Azores islands groups. Geological Society of America Bulletin 84, 633-648.

Self, S., 1976. The recent volcanology of Terceira, Azores. Journal of the Geological Society of London 132, 645-666.

Self, S., Gunn, B.M., 1976. Petrology, volume and age relations of alkaline and saturated peralkaline volcanics from Terceira, Azores. Contributions to Mineralogy and Petrology 54, 293-313.

Silveira, G., Stutzmann, E., Davaille, A., Montagner, J.-P., Mendes-Victor, L., Sebai, A., 2006. Azores hotspot signature in the upper mantle. Journal of Volcanology and Geothermal Research 156, 23-34.

Silveira, G., Vinnik, L., Stutzmann, E., Farra, V., Kiselev, S., Morais, I., 2010. Stratification of the Earth beneath the Azores from $\mathrm{P}$ and $\mathrm{S}$ receiver functions. Earth and Planetary Science Letters 229, 91-103.

Sobolev, A.V., Hofmann, A.W., Sobolev, S.V., Nikogosian, I.K., 2005. An olivine-free mantle source of Hawaiian shield basalts. Nature 434, 590-597.

Sridhar, D.Y., Ray, D., 2003. Structure, tectonic and petrology of mid-oceanic ridges and the Indian scenario. Current Science 85 (3), 277-283.

Stracke, A., Bizimis, M., Salters, V.J., 2003. Recycling oceanic crust: quantitative constraints. Geochemistry, Geophysics, Geosystems 4 (3), 8003. doi:10.1029/ 2001 GC000223.

Sun, S.S., McDonough, W.F., 1989. Chemical and isotopic systematics of oceanic basalts: implications for mantle composition and processes. In: Saunders, A.D., Norry, M.J. (Eds.), Magmatism in the Ocean Basins: Geological Society of London, Special Publication, 42, pp. 313-345.

Tappe, S., 2004. Mesozoic mafic alkaline magmatism of southern Scandinavia. Contributions to Mineralogy and Petrology 148, 312-334.

Tappe, S., Foley, S.F., Stracke, A., Romer, R.L., Kjarsgaard, B.A., Heaman, L.M., Joyce, N., 2007. Craton reactivation on the Labrador Sea margins: ${ }^{40} \mathrm{Ar} /{ }^{39} \mathrm{Ar}$ age and $\mathrm{Sr}-\mathrm{Nd}-$ $\mathrm{Hf}-\mathrm{Pb}$ isotope constraints from alkaline and carbonatite intrusives. Earth and Planetary Science Letters 256, 433-454.

Toplis, M.J., Carroll, M.R., 1995. An experimental study of the influence of oxygen fugacity on Fe-Ti oxide stability, phase relations, and mineral-melt equilibria in ferro-basaltic systems. Journal of Petrology 36, 1137-1170.

Turner, S., Hawkesworth, C., Rogers, N., King, P., 1997. U-Th isotope disequilibria and ocean islands basalt generation in the Azores. Chemical Geology 139, 145-164.

Vogt, P.R., Jung, W.Y., 2004. The Terceira Rift as a hyper-slow, hotspot-dominated oblique spreading axis: a comparison with other slow-spreading plate boundaries. Earth and Planetary Science Letters 218, 77-90. 
Weaver, B.L., 1991. The origin of oceanic island basalt endmember compositions: trace element and isotopic constraints. Earth and Planetary Science Letters 104, 381-397.

Weis, D., Kieffer, B., Maerschalk, C., Barling, J., De Jong, J., Williams, G., Hanano, D. Pretorius, W., Mattielli, N., Scoates, J.S., Goolaerts, A., Friedman, R., Mahoney, J.B., 2006. High-precision isotopic characterization of USGS reference materials by TIMS and MC-ICP-MS. Geochemistry, Geophysics, Geosystems 7, Q08006. doi:10.1029/ 2006 GC001283.

White, W.W., Tapia, M.D., Schilling, J.G., 1979. The petrology and geochemistry of the Azores Islands. Contributions to Mineralogy and Petrology 69, 201-213.

Widom, E., Shirey, S.B., 1996. Os isotope systematics in the Azores: implications for mantle plume sources. Earth and Planetary Science Letters 142, 451-465.

Widom, E., Carlson, R.W., Gill, J.B., Schmincke, H.-U., 1997. Th-Sr-Nd-Pb isotope and trace element evidence for the origin of the São Miguel, Azores, enriched mantle source. Chemical Geology 140, 49-68.
Woodhead, J.D., 1996. Extreme HIMU in an oceanic setting: the geochemistry of Mangaia island (Polynesia), and temporal evolution of the Cook-Austral hot spot Journal of Volcanology and Geothermal Research 72, 1-19.

Yang, T., Shen, Y., Van der Lee, S., Solomon, S.C., Hung, S.C., 2006. Upper mantle structure beneath the Azores hotspot from finite-frequency seismic tomography. Earth and Planetary Science Letters 250, 11-26.

Zbyszewski, G., Cândido de Medeiros, A., Veiga Ferreira, O., Torre de Assunção, C., 1971 Carta Geológica de Portugal na escala 1:50,000: ilha Terceira. Notícia explicativa. Serviços Geológicos de Portugal. 43 pp.

Zindler, A., Hart, S., 1986. Chemical geodynamics. Annual Review of Earth and Planetary Sciences 14, 493-571. 